\title{
Some Existence and Dependence Criteria of Solutions to a Fractional Integro-Differential Boundary Value Problem via the Generalized Gronwall Inequality
}

\author{
Shahram Rezapour ${ }^{1,2,+}\left(\mathbb{D}\right.$, Sotiris K. Ntouyas ${ }^{3,4,+} \mathbb{D}$, Abdelkader Amara ${ }^{5,+}$, Sina Etemad ${ }^{2,+}$ \\ and Jessada Tariboon ${ }^{6, *,+}$ (D) \\ 1 Department of Medical Research, China Medical University Hospital, China Medical University, \\ Taichung 404, Taiwan; rezapourshahram@yahoo.ca \\ 2 Department of Mathematics, Azarbaijan Shahid Madani University, Tabriz 51368, Iran; \\ sina.etemad@azaruniv.ac.ir or sina.etemad@gmail.com \\ 3 Department of Mathematics, University of Ioannina, 45110 Ioannina, Greece; sntouyas@uoi.gr \\ 4 Nonlinear Analysis and Applied Mathematics (NAAM)-Research Group, Department of Mathematics, \\ Faculty of Science, King Abdulaziz University, P.O. Box 80203, Jeddah 21589, Saudi Arabia \\ 5 Laboratory of Applied Mathematics, University of Kasdi Merbah Ouargla, Ouargla 30000, Algeria; \\ amara.abdelkader@univ-ouargla.dz \\ 6 Intelligent and Nonlinear Dynamic Innovations Research Center, Department of Mathematics, \\ Faculty of Applied Science, King Mongkut's University of Technology North Bangkok, \\ Bangkok 10800, Thailand \\ * Correspondence: jessada.t@sci.kmutnb.ac.th \\ + These authors contributed equally to this work.
}

Citation: Rezapour, S.; Ntouyas, S.K.; Amara, A.; Etemad, S.; Tariboon, J. Some Existence and Dependence Criteria of Solutions to a Fractional Integro-Differential Boundary Value Problem via the Generalized Gronwall Inequality. Mathematics 2021, 9, 1165. https://doi.org/ $10.3390 /$ math 9111165

Academic Editor: Dorota Mozyrska

Received: 11 April 2021

Accepted: 19 May 2021

Published: 21 May 2021

Publisher's Note: MDPI stays neutral with regard to jurisdictional claims in published maps and institutional affiliations.

Copyright: (c) 2020 by the authors. Licensee MDPI, Basel, Switzerland. This article is an open access article distributed under the terms and conditions of the Creative Commons Attribution (CC BY) license (https:// creativecommons.org/licenses/by/ $4.0 /)$.
Abstract: The main intention of the present research study is focused on the analysis of a Caputo fractional integro-differential boundary problem (CFBVP) in which the right-hand side of supposed differential equation is represented as a sum of two nonlinear terms. Under the integro-derivative boundary conditions, we extract an equivalent integral equation and then define new operators based on it. With the help of three distinct fixed-point theorems attributed to Krasnosel'skii, Leray-Schauder, and Banach, we investigate desired uniqueness and existence results. Additionally, the dependence criterion of solutions for this CFBVP is checked via the generalized version of the Gronwall inequality. Next, three simulative examples are designed to examine our findings based on the procedures applied in the theorems.

Keywords: caputo derivative; fixed point; integral equation; generalized Gronwall inequality

\section{Introduction}

On a large scale, it is specified that the hereditary aspects and the memory of different processes and materials are predictable with the help of some models under the fractional operators. In this regard, differential equations with arbitrary-order derivatives have recently proven to be an efficient tool in the modeling of a vast variety of phenomena in several branches of science [1-4]. To be more precise, one can view the deep effectiveness of this category of fractional equation in the modeling of some areas involving relaxation vibrations, viscoelasticity, electrochemistry, signal processing, diffusion procedures, etc. (see [5-12] and references therein). Since theoretical findings and outcomes can support the arrival at a profound understanding for the arbitrary-order models, a large number of mathematicians have tended to discuss the existence and dependence aspects of solutions of various structures of fractional equations (see [13-25]). In [26] a discrete Gronwall inequality was introduced to provide a stability bound. In addition to these, some formulations as integro-differential equations have recently been analyzed by researchers [27-33]. 
Zhao, Huang et al. [34] carried out a survey on the existence aspects of solutions for a multipoint nonlinear integro-differential boundary value problem (FBVP) as

$$
\left\{\begin{array}{l}
\mathcal{R}_{\mathfrak{D}_{0^{+}}^{\sigma}} \omega(s)+\hbar\left(s, \omega(s), \omega^{\prime}(s), \ldots, \omega^{(k-2)}(s), \mathbb{A}_{1} \omega(s), \mathbb{A}_{2} \omega(s)\right)=0, \quad(0 \leq s \leq 1), \\
\omega(0)=\omega^{\prime}(0)=\cdots=\omega^{(k-2)}(0)=0, \\
\omega^{(k-2)}(1)=\sum_{j=1}^{m-2} K_{j} \omega^{(k-2)}\left(\xi_{j}\right), \quad\left(K_{j}>0,0<\xi_{1}<\xi_{2}<\cdots<\xi_{m-2}<1\right),
\end{array}\right.
$$

so that $k-1<\sigma<k$, and for functions $\mathbb{B}_{1} \in \mathcal{C}\left(M=\{(s, \eta) \in[0,1] \times[0,1]: s \geq \eta\}, \mathbb{R}^{>0}\right)$ and $\mathbb{B}_{2} \in \mathcal{C}\left([0,1] \times[0,1], \mathbb{R}^{>0}\right)$, we have

$$
\mathbb{A}_{1} \omega(s)=\int_{0}^{s} \mathbb{B}_{1}(s, \eta) \omega(\eta) \mathrm{d} \eta, \quad \mathbb{A}_{2} \omega(s)=\int_{0}^{1} \mathbb{B}_{2}(s, \eta) \omega(\eta) \mathrm{d} \eta .
$$

In that work, they utilized strict-set contractions on the structures named cones to extract criteria of existence [34].

In 2016, Ahmad, Ntouyas, and Tariboon [35] implemented an analytical research study to check some required conditions for proving the existence of possible solutions of the hybrid integro-inclusion FBVP given by

$$
\mathcal{C}_{\mathfrak{D}_{0^{+}}^{\sigma}}\left[\frac{\omega(s)-\sum_{l=1}^{k} \mathcal{R}^{\mathcal{I}_{0^{+}}^{\sigma_{l}} \hbar_{l}^{*}(s, \omega(s))}}{\phi^{*}(s, \omega(s))}\right] \in \mathfrak{P}(s, \omega(s)), \quad(0 \leq s \leq 1),
$$

such that $\omega(0)=\varphi(s)$ and $\omega(1)=b \in \mathbb{R}$ and ${ }^{\mathcal{C}} \mathfrak{D}_{0^{+}}^{\sigma}$ and ${ }^{\mathcal{R}} \mathcal{I}_{0^{+}}$stand for the fractional Caputo and Riemann-Liouville operators of orders $\sigma \in[1,2)$ and $\iota>0$ with $\iota \in\left\{\sigma_{1}, \sigma_{2}, \ldots, \sigma_{k}\right\}$. In 2018, Bazgir and Ghazanfari [36] proceeded to obtain some desired conditions for which the new category of nonlinear Caputo integro-differential FBVP

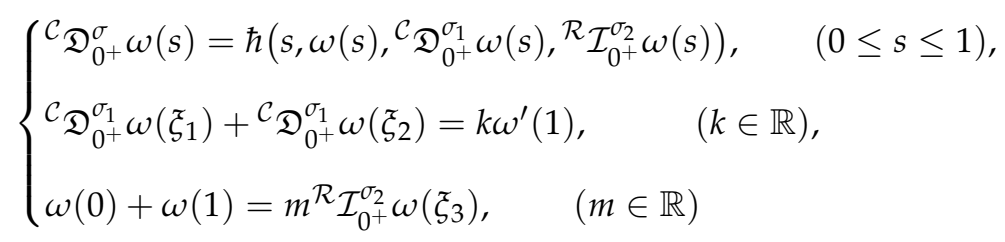

has at least one solution on the relevant domain; in this structure, it is supposed that $\sigma \in(1,2), \sigma_{1} \in(0,1)$ with $\sigma>\sigma_{1}+1, \sigma_{2}>0$ and $\xi_{1}, \xi_{2}, \xi_{3} \in(0,1)$.

By taking the existing ideas of some of the above articles, we turn to discuss on a newly designed extended framework of the Caputo fractional mixed integro-differential equation with fractional integral conditions

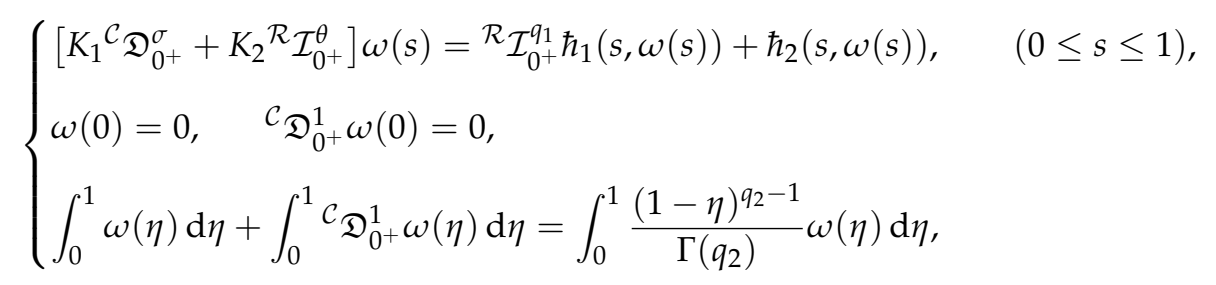

where $\sigma \in(2,3), \theta, q_{1}, q_{2} \geq 0$ and $K_{1}, K_{2} \in \mathbb{R}^{+}$with $K_{1} \neq 0$. Two symbols $\mathcal{C}_{\mathfrak{D}_{0^{+}}}^{(\cdot)}$ and ${ }^{\mathcal{R}} \mathcal{I}_{0^{+}}^{(\cdot)}$ denote the Caputo fractional derivative (CF-derivative) and the Riemann-Liouville fractional integral (RLF-integral) of different fractional orders. Additionally, both functions $\hbar_{1}$ and $\hbar_{2}$ are supposed to be continuous on $[0,1] \times \mathbb{R}$ with real values. It is a considerable issue that the researchers concentrate on the fact that the proposed Caputo fractional integrodifferential boundary problem (CFBVP) has both aspects of generality and uniqueness in its structure. In fact, the existing construction for the aforementioned integro-differential 
CFBVP (1) has two different derivatives in the Caputo settings along with three distinct integrals of the RLF-type. The right-hand side of this problem is as a sum of two nonlinear terms under functions $\hbar_{1}$ and $\hbar_{2}$. This composition of the given FBVP involves several types of special case of the fractional differential equations. It is sufficient that we consider specific values for parameters of the given CFBVP to derive some new fractional models arising in real-life phenomena or processes. For instance, if we consider $\theta=q_{1}=0, K_{2}=1$, and $\hbar_{2} \equiv 0$, then for $0<\mu<1$ we have the fractional pantograph differential equation

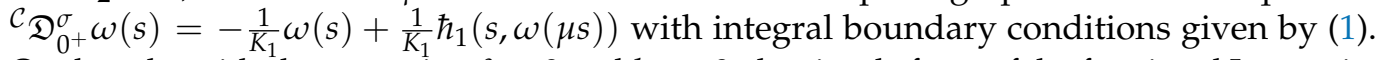
On the other side, by assuming $\theta=0$ and $\hbar_{2} \equiv 0$, the simple form of the fractional Langevin integro-differential equation is derived as $\left[K_{1} \mathcal{C}_{\mathfrak{D}_{0^{+}}^{\sigma}}+K_{2}\right] \omega(s)={ }^{\mathcal{R}} \mathcal{I}_{0^{+}}^{q_{1}} \hbar_{1}(s, \omega(s))$. Therefore, we can extract other simple forms of well-known boundary value problems, and so all results, theorems, and examples of this manuscript are applicable for such special actual systems. Finally, we implicitly state that the aforementioned type of integro-differential CFBVP equipped with such conditions has not been discussed in the existing works, and it has an abstract and general format. In this direction, we use familiar and famous analytical methods to establish the required criteria which prove the existence theorems for the proposed integro-differential CFBVP (1). In the sequel, we analyze the dependence of solutions to a special case of integro-differential CFBVP (1).

The scheme of the current research is arranged as follows. In Section 2, some basic notions on fractional calculus (FC) are collected. Section 3 proves essential theorems which guarantee the existence of possible solutions for the suggested integro-differential CFBVP (1). Additionally, by considering the generalized version of the Gronwall inequality, the dependence of solutions to a special case of the integro-differential CFBVP (1) is analyzed. With the help of some special numerical examples in Section 4 , we validate our theoretical outcomes according to methods implemented in theorems.

\section{Auxiliary Preliminaries}

This section is assigned to recall some basic notions and definitions in relation to fractional calculus.

Definition 1 ([37,38]). Let $\sigma>0$. The Riemann-Liouville fractional integral (RLF-integral) for a real-valued continuous function $\omega$ on $[a, b]$ is given by

$$
\mathcal{R}_{\mathcal{I}_{0^{+}}^{\sigma} \omega(s)}=\frac{1}{\Gamma(\sigma)} \int_{0}^{s}(s-\eta)^{\sigma-1} \omega(\eta) \mathrm{d} \eta
$$

provided that the finite values exist for the right-hand side integral.

Definition 2 ([37,38]). Let $k-1<\sigma<k$ or $k=1+[\sigma]$. The Caputo fractional derivative (CF-derivative) of an existing function $\omega \in \mathcal{C}_{\mathbb{R}}^{(k)}([a, b])$ is defined as follows:

$$
\mathcal{C}_{\mathfrak{D}_{0^{+}}^{\sigma} \omega(s)}=\frac{1}{\Gamma(k-\sigma)} \int_{0}^{s}(s-\eta)^{k-\sigma-1} \omega^{(k)}(\eta) \mathrm{d} \eta
$$

such that the existing integral in the above equation involves finite values.

Lemma 1 ([4]). Let $\sigma_{2}>\sigma_{1}>0$ and $\omega \in \mathcal{L}^{1}([a, b])$. Then, for all $s \in[a, b]$, we have

(i) ${ }^{R} \mathcal{I}_{0^{+}}^{\sigma_{1}} \mathcal{R} \mathcal{I}_{0^{+}}^{\sigma_{2}} \omega(s)={ }^{R} \mathcal{I}_{0^{+}}^{\sigma_{1}+\sigma_{2}} \omega(s)$;

(ii) $\mathcal{C}_{\mathfrak{D}_{0^{+}}^{\sigma_{1}}} \mathcal{R}_{\mathcal{I}_{0^{+}}^{\sigma_{2}}} \omega(s)={ }^{\mathcal{R}} \mathcal{I}_{0^{+}}^{\sigma_{2}-\sigma_{1}} \omega(s)$.

In [39], we may observe that the general series solution about the given differential equation $C_{\mathfrak{D}_{0^{+}}^{\sigma}}^{\sigma} \omega(s)=0$ equals to $\omega(s)=c_{0}+c_{1} s+c_{2} s^{2}+\cdots+c_{k-1} s^{k-1}$ and

$$
\mathcal{R}_{\mathcal{I}_{0^{+}}^{\sigma}}\left(\mathcal{C}_{\mathfrak{D}_{0^{+}}^{\sigma}}^{\sigma} \omega(s)\right)=\omega(s)+\sum_{m=0}^{k-1} c_{m} s^{m}=\omega(s)+c_{0}+c_{1} s+c_{2} s^{2}+\cdots+c_{k-1} s^{k-1}
$$


holds such that $c_{0}, \ldots, c_{k-1} \in \mathbb{R}$ and $k=1+[\sigma]$.

To arrive at the main aims for proving the conditions of the existence of solutions of (1) here, three fixed-point results in relation to this purpose are recalled.

Theorem 1 ([40]). By assuming $Y \neq \varnothing$ as a closed, convex, and bounded set contained in the Banach space $X$, the following assertions hold for $\hbar_{1}, \hbar_{2}: Y \rightarrow X$ :

(th1) $\hbar_{1} \omega_{1}+\hbar_{2} \omega_{2} \in Y$ for $\omega_{1}, \omega_{2} \in Y$;

(th2) $\hbar_{1}$ is a contraction;

(th3) $\hbar_{2}$ possesses both properties of continuity and compactness.

Then, a member $\omega^{*} \in Y$ exists such that $\omega^{*}=\hbar_{1} \omega^{*}+\hbar_{2} \omega^{*}$.

Theorem 2 ([41]). Consider $X$ and $Y$ as a Banach space and a convex closed subset of this space, respectively. Further, let $\mathbb{V}$ be an open set contained in $Y$ with $0 \in \mathbb{V}$ and $\hbar: \overline{\mathbb{V}} \rightarrow Y$ possesses both properties of continuity and compactness. In that case, either:

(th4) $\hbar$ involves a fixed point in $\overline{\mathbb{V}}$; or

(th5) $\omega^{*} \in \partial \mathbb{V}$ and $\hat{a} \in(0,1)$ exist subject to $\omega^{*}=\hat{a} \hbar\left(\omega^{*}\right)$.

Theorem 3 ([42]). The set $X$ is considered as a Banach space and further $\hbar: Y \rightarrow Y$ is regarded as a contraction map on the closed set $Y \subset X$. Then $\hbar$ contains a fixed point uniquely in $Y$.

Theorem 4 ([43,44]). (The generalized Gronwall inequality) Let $u:[0, T] \rightarrow \mathbb{R}^{+}$be locally integrable for $T \leq \infty$ and $\psi:[0, T] \rightarrow \mathbb{R}^{+}$be nondecreasing and continuous with $\psi(s) \leq M \neq 0$ and $\hat{\omega}:[0, T] \rightarrow \mathbb{R}^{+}$be a locally integrable mapping with

$$
\hat{\omega}(s) \leq u(s)+\psi(s) \int_{0}^{s}(s-\eta)^{\sigma-1} \hat{\omega}(\eta) \mathrm{d} \eta
$$

such that $\sigma>0$. Then

$$
\hat{\omega}(s) \leq u(s)+\int_{0}^{s} \sum_{m=1}^{\infty}\left[\frac{(\psi(s) \Gamma(\sigma))^{m}}{\Gamma(m \sigma)}(s-\eta)^{m \sigma-1} u(\eta)\right] \mathrm{d} \eta, \quad(s \in[0, T]) .
$$

\section{Main Theorems}

By $X=\mathcal{C}_{\mathbb{R}}([0,1])$ we mean the collection of all given continuous maps on $[0,1]$ subject to real values. We can easily confirm that $X$ will be a Banach space whenever we regard a sup norm on it as $\|\omega\|=\sup _{s \in[0,1]}|\omega(s)|$ for $\omega \in X$. Now, a structural proposition is provided to specify the formulation of the possible solutions of an integral equation arising from the proposed integro-differential CFBVP (1).

Proposition 1. Let $\hbar \in X$ and regard constants $\sigma \in(2,3), q_{2}, \theta>0$ and $K_{1}, K_{2} \in \mathbb{R}$ with $K_{1} \neq 0$. In that case, the function $\omega^{*}$ is to be known as a solution for the CFBVP constructed by

$$
\left\{\begin{array}{l}
{\left[K_{1} \mathcal{C}_{\mathfrak{D}_{0^{+}}}^{\sigma}+K_{2}{ }^{\mathcal{R}} \mathcal{I}_{0^{+}}^{\theta}\right] \omega(s)=\hbar(s), \quad(0 \leq s \leq 1),} \\
\omega(0)=0, \quad \mathcal{C}_{\mathfrak{D}_{0^{+}}}^{1} \omega(0)=0, \\
\int_{0}^{1} \omega(\eta) \mathrm{d} \eta+\int_{0}^{1} \mathcal{C}_{\mathfrak{D}_{0^{+}}}^{1} \omega(\eta) \mathrm{d} \eta=\int_{0}^{1} \frac{(1-\eta)^{q_{2}-1}}{\Gamma\left(q_{2}\right)} \omega(\eta) \mathrm{d} \eta,
\end{array}\right.
$$


given by the RLF-integral equation

$$
\begin{aligned}
\omega(s)= & \frac{1}{K_{1}} \int_{0}^{s} \frac{(s-\eta)^{\sigma-1}}{\Gamma(\sigma)} \hbar(\eta) \mathrm{d} \eta-\frac{K_{2}}{K_{1}} \int_{0}^{s} \frac{(s-\eta)^{\sigma+\theta-1}}{\Gamma(\sigma+\theta)} \omega(\eta) \mathrm{d} \eta \\
& +\frac{s^{2}}{\xi K_{1}}\left[\int_{0}^{1} \frac{(1-\eta)^{\sigma+q_{2}-1}}{\Gamma\left(\sigma+q_{2}\right)} \hbar(\eta) \mathrm{d} \eta-\int_{0}^{1} \int_{0}^{\eta} \frac{(\eta-r)^{\sigma-1}}{\Gamma(\sigma)} \hbar(r) \mathrm{d} r \mathrm{~d} \eta\right. \\
& \left.-\int_{0}^{1} \int_{0}^{\eta} \frac{(\eta-r)^{\sigma-2}}{\Gamma(\sigma-1)} \hbar(r) \mathrm{d} r \mathrm{~d} \eta\right] \\
& +\frac{s^{2} K_{2}}{\xi K_{1}}\left[-\int_{0}^{1} \frac{(1-\eta)^{\sigma+\theta+q_{2}-1}}{\Gamma\left(\sigma+\theta+q_{2}\right)} \omega(\eta) \mathrm{d} \eta+\int_{0}^{1} \int_{0}^{\eta} \frac{(\eta-r)^{\sigma+\theta-1}}{\Gamma(\sigma+\theta)} \omega(r) \mathrm{d} r \mathrm{~d} \eta\right. \\
& \left.+\int_{0}^{1} \int_{0}^{\eta} \frac{(\eta-r)^{\sigma+\theta-2}}{\Gamma(\sigma+\theta-1)} \omega(r) \mathrm{d} r \mathrm{~d} \eta\right]
\end{aligned}
$$

in which it is assumed that

$$
\xi:=\left|\frac{4 \Gamma\left(q_{2}+3\right)-6}{3 \Gamma\left(q_{2}+3\right)}\right| \neq 0
$$

Proof. In the first stage, we proceed to assume that $\omega^{*}$ is a solution of the CFBVP (2). So, we get

$$
\left[K_{1}{ }^{\mathcal{C}} \mathfrak{D}_{0^{+}}^{\sigma}+K_{2}{ }^{\mathcal{R}} \mathcal{I}_{0^{+}}^{\theta}\right] \omega^{*}(s)=\hbar(s)
$$

and hence

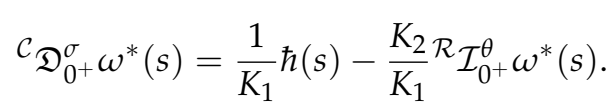

We take the RLF-integral of order $\sigma$ on two sides of the above relation and we arrive at

$$
\omega^{*}(s)=\frac{1}{K_{1}} \int_{0}^{s} \frac{(s-\eta)^{\sigma-1}}{\Gamma(\sigma)} \hbar(\eta) \mathrm{d} \eta-\frac{K_{2}}{K_{1}} \int_{0}^{s} \frac{(s-\eta)^{\sigma+\theta-1}}{\Gamma(\sigma+\theta)} \omega^{*}(\eta) \mathrm{d} \eta+c_{0}+c_{1} s+c_{2} s^{2}
$$

in which $c_{0}, c_{1}, c_{2} \in \mathbb{R}$ are real numbers that we have to find. It is quickly understood that $c_{0}=0$ by virtue of the condition $\omega^{*}(0)=0$ and (6). In addition, by regarding

$$
\mathcal{C}_{\mathfrak{D}_{0^{+}}}^{1} \omega^{*}(s)=\frac{1}{K_{1}} \int_{0}^{s} \frac{(s-\eta)^{\sigma-2}}{\Gamma(\sigma-1)} \hbar(\eta) \mathrm{d} \eta-\frac{K_{2}}{K_{1}} \int_{0}^{s} \frac{(s-\eta)^{\sigma+\theta-2}}{\Gamma(\sigma+\theta-1)} \omega^{*}(\eta) \mathrm{d} \eta+c_{1}+2 c_{2} s
$$

and by virtue of the second condition ${ }^{\mathcal{C}} \mathfrak{D}_{0^{+}}^{1} \omega^{*}(0)=0$, we get $c_{1}=0$. On the other side, by keeping in mind the properties of the RLF-integral, we have

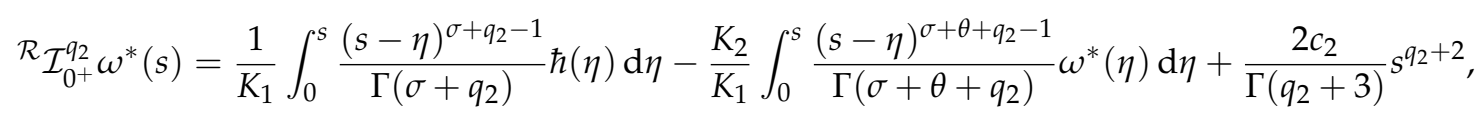

for $q_{2}>0$. Eventually, the mixed integral boundary condition

$$
\int_{0}^{1} \omega^{*}(\eta) \mathrm{d} \eta+\int_{0}^{1} \mathcal{C}_{\mathfrak{D}_{0^{+}}^{1}} \omega^{*}(\eta) \mathrm{d} \eta=\int_{0}^{1} \frac{(1-\eta)^{q_{2}-1}}{\Gamma\left(q_{2}\right)} \omega^{*}(\eta) \mathrm{d} \eta
$$


implies that we arrive at the following:

$$
\begin{aligned}
c_{2}= & \frac{1}{\xi K_{1}}\left[\int_{0}^{1} \frac{(1-\eta)^{\sigma+q_{2}-1}}{\Gamma\left(\sigma+q_{2}\right)} \hbar(\eta) \mathrm{d} \eta-\int_{0}^{1} \int_{0}^{\eta} \frac{(\eta-r)^{\sigma-1}}{\Gamma(\sigma)} \hbar(r) \mathrm{d} r \mathrm{~d} \eta\right. \\
& \left.-\int_{0}^{1} \int_{0}^{\eta} \frac{(\eta-r)^{\sigma-2}}{\Gamma(\sigma-1)} \hbar(r) \mathrm{d} r \mathrm{~d} \eta\right]+\frac{K_{2}}{\xi K_{1}}\left[-\int_{0}^{1} \frac{(1-\eta)^{\sigma+\theta+q_{2}-1}}{\Gamma\left(\sigma+\theta+q_{2}\right)} \omega^{*}(\eta) \mathrm{d} \eta\right. \\
& \left.+\int_{0}^{1} \int_{0}^{\eta} \frac{(\eta-r)^{\sigma+\theta-1}}{\Gamma(\sigma+\theta)} \omega^{*}(r) \mathrm{d} r \mathrm{~d} \eta+\int_{0}^{1} \int_{0}^{\eta} \frac{(\eta-r)^{\sigma+\theta-2}}{\Gamma(\sigma+\theta-1)} \omega^{*}(r) \mathrm{d} r \mathrm{~d} \eta\right]
\end{aligned}
$$

so that $\xi \neq 0$ is introduced as (4). Consequently, we insert all three obtained constants $c_{0}, c_{1}$, and $c_{2}$ into (6). The resulting relation shows that $\omega^{*}$ satisfies the RLF-integral Equation (3) and thus $\omega^{*}$ will be a solution for it. This finishes the argument.

Considering Proposition 1 and the proposed nonlinear integro-differential CFBVP (1), we formulate the nonlinear operator $\mathbb{T}: X \rightarrow X$ as

$$
\begin{aligned}
& (\mathbb{T} \omega)(s)=\frac{1}{K_{1}} \mathcal{R}_{0^{+}}^{\sigma+q_{1}} \hbar_{1}(s, \omega(s))+\frac{1}{K_{1}} \mathcal{R}_{\mathcal{I}^{+}}^{\sigma} \hbar_{2}(s, \omega(s))-\frac{K_{2}}{K_{1}} \int_{0}^{s} \frac{(s-\eta)^{\sigma+\theta-1}}{\Gamma(\sigma+\theta)} \omega(\eta) \mathrm{d} \eta \\
& +\frac{s^{2}}{\xi K_{1}}\left[\mathcal{R}_{\mathcal{I}^{+}}^{\sigma+q_{2}+q_{1}} \hbar_{1}(1, \omega(1))+{ }^{\mathcal{R}} \mathcal{I}_{0^{+}}^{\sigma+q_{2}} \hbar_{2}(1, \omega(1))-\int_{0}^{1} \mathcal{R} \mathcal{I}_{0^{+}}^{\sigma+q_{1}} \hbar_{1}(\eta, \omega(\eta)) \mathrm{d} \eta\right.
\end{aligned}
$$

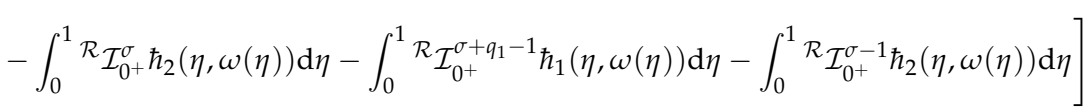

$$
\begin{aligned}
& +\frac{s^{2} K_{2}}{\xi K_{1}}\left[-\int_{0}^{1} \frac{(1-\eta)^{\sigma+\theta+q_{2}-1}}{\Gamma\left(\sigma+\theta+q_{2}\right)} \omega(\eta) \mathrm{d} \eta+\int_{0}^{1} \int_{0}^{\eta} \frac{(\eta-r)^{\sigma+\theta-1}}{\Gamma(\sigma+\theta)} \omega(r) \mathrm{d} r \mathrm{~d} \eta\right. \\
& \left.+\int_{0}^{1} \int_{0}^{\eta} \frac{(\eta-r)^{\sigma+\theta-2}}{\Gamma(\sigma+\theta-1)} \omega(r) \mathrm{d} r \mathrm{~d} \eta\right]
\end{aligned}
$$

for $\omega \in X$ and $0 \leq s \leq 1$. We give three constants which we shall employ later:

$$
\begin{aligned}
\tilde{\Delta}^{(1)} & :=\frac{K_{2}}{K_{1} \Gamma(\sigma+\theta+1)}+\frac{K_{2}}{\xi K_{1}}\left[\frac{1}{\Gamma\left(\sigma+\theta+q_{2}+1\right)}+\frac{1}{\Gamma(\sigma+\theta+2)}+\frac{1}{\Gamma(\sigma+\theta+1)}\right], \\
\tilde{\Delta}^{(2)} & :=\frac{1}{K_{1} \Gamma(\sigma+1)}+\frac{1}{\xi K_{1}}\left[\frac{1}{\Gamma\left(\sigma+q_{2}+1\right)}+\frac{1}{\Gamma(\sigma+2)}+\frac{1}{\Gamma(\sigma+1)}\right], \\
\tilde{\Delta}^{(3)} & :=\frac{1}{K_{1} \Gamma\left(\sigma+q_{1}+1\right)}+\frac{1}{\xi K_{1}}\left[\frac{1}{\Gamma\left(\sigma+q_{2}+q_{1}+1\right)}+\frac{1}{\Gamma\left(\sigma+q_{1}+2\right)}+\frac{1}{\Gamma\left(\sigma+q_{1}+1\right)}\right] .
\end{aligned}
$$

At this moment, we can begin our argument for deriving existence factors for the existing nonlinear integro-differential CFBVP (1). To implement the procedure, we use the famous fixed-point result in the functional analysis due to Krasnosel'skii.

Theorem 5. Both single-valued maps $\hbar_{j}:[0,1] \times X \rightarrow X(j=1,2)$ are regarded to be continuous and further to possess the following specifications:

$(\mathbb{C} 1)$ A number $\hat{L}^{*}>0$ exists provided that for all $\omega_{1}, \omega_{2} \in X$ and $0 \leq s \leq 1$, the Lipschitz property $\left|\hbar_{2}\left(s, \omega_{1}(s)\right)-\hbar_{2}\left(s, \omega_{2}(s)\right)\right| \leq \hat{L}^{*}\left|\omega_{1}-\omega_{2}\right|$ is valid; 
$(\mathbb{C} 2)$ A continuous function $\Phi$ on $[0,1]$ with real values exists for which the boundedness property $\left|\hbar_{1}(s, \omega(s))\right| \leq \Phi(s)$ holds for $0 \leq s \leq 1$ and for each $\omega \in X$.

Then the given nonlinear integro-differential CFBVP (1) includes at least one solution on $[0,1]$ if $\tilde{\Delta}^{(1)}+\hat{L}^{*} \tilde{\Delta}^{(2)}<1$ exists, where $\tilde{\Delta}^{(j)}(j=1,2)$ are available in (8).

Proof. We introduce $\|\Phi\|=\sup _{s \in[0,1]}|\Phi(s)|$ and $\mathbb{B}_{\tilde{r}}:=\{\omega \in X:\|\omega\| \leq \tilde{r}\}$ with

$$
\tilde{r} \geq \frac{\|\Phi\| \tilde{\Delta}^{(3)}+\hat{\mathbb{H}}_{*} \tilde{\Delta}^{(2)}}{1-\left(\tilde{\Delta}^{(1)}+\hat{L}^{*} \tilde{\Delta}^{(2)}\right)}
$$

where $\hat{\mathbb{H}}_{*}:=\sup _{s \in[0,1]}\left|\hbar_{2}(s, 0)\right|$ and $\tilde{\Delta}^{(j)}(j=1,2,3)$ are represented in (8). According to the previous information, the above ball $\mathbb{B}_{\tilde{r}}$ is a nonempty closed bounded convex set contained in the Banach space $X$. Along with this, we point out an operator $\mathbb{T}: X \rightarrow X$ as (7). By Proposition 1, it is apparent that the collection of the fixed points of $\mathbb{T}$ is the same as the possible solutions of the nonlinear integro-differential CFBVP (1). To proceed in proving this issue, we give two operators, namely, $\mathbb{T}_{1}$ and $\mathbb{T}_{2}$, from $\mathbb{B}_{\tilde{r}}$ to $X$ which have the structures

$$
\begin{aligned}
& \mathbb{T}_{1} \omega(s)=\frac{1}{K_{1}} \mathcal{R}_{\mathcal{I}^{+}}^{\sigma} \hbar_{2}(s, \omega(s))-\frac{K_{2}}{K_{1}} \int_{0}^{s} \frac{(s-\eta)^{\sigma+\theta-1}}{\Gamma(\sigma+\theta)} \omega(\eta) \mathrm{d} \eta \\
& +\frac{s^{2}}{\xi K_{1}}\left[{ }^{\mathcal{R}} \mathcal{I}_{0^{+}}^{\sigma+q_{2}} \hbar_{2}(1, \omega(1))-\int_{0}^{1}{ }^{\mathcal{R}} \mathcal{I}_{0^{+}}^{\sigma} \hbar_{2}(\eta, \omega(\eta)) \mathrm{d} \eta-\int_{0}^{1}{ }^{\mathcal{R}} \mathcal{I}_{0^{+}}^{\sigma-1} \hbar_{2}(\eta, \omega(\eta)) \mathrm{d} \eta\right] \\
& +\frac{s^{2} K_{2}}{\xi K_{1}}\left[-\int_{0}^{1} \frac{(1-\eta)^{\sigma+\theta+q_{2}-1}}{\Gamma\left(\sigma+\theta+q_{2}\right)} \omega(\eta) \mathrm{d} \eta+\int_{0}^{1} \int_{0}^{\eta} \frac{(\eta-r)^{\sigma+\theta-1}}{\Gamma(\sigma+\theta)} \omega(r) \mathrm{d} r \mathrm{~d} \eta\right. \\
& \left.+\int_{0}^{1} \int_{0}^{\eta} \frac{(\eta-r)^{\sigma+\theta-2}}{\Gamma(\sigma+\theta-1)} \omega(r) \mathrm{d} r \mathrm{~d} \eta\right]
\end{aligned}
$$

and

$$
\begin{aligned}
\mathbb{T}_{2} \omega(s)= & \frac{1}{K_{1}}{ }^{\mathcal{R}} \mathcal{I}_{0^{+}}^{\sigma+q_{1}} \hbar_{1}(s, \omega(s))+\frac{s^{2}}{\xi K_{1}}\left[\mathcal{R}_{\mathcal{I}_{0^{+}}^{\sigma+q_{2}+q_{1}} \hbar_{1}(1, \omega(1))-\int_{0}^{1}{ }^{\mathcal{R}} \mathcal{I}_{0^{+}}^{\sigma+q_{1}} \hbar_{1}(\eta, \omega(\eta)) \mathrm{d} \eta}\right. \\
& \left.-\int_{0}^{1} \mathcal{R}_{\mathcal{I}_{0^{+}}}^{\sigma+q_{1}-1} \hbar_{1}(\eta, \omega(\eta)) \mathrm{d} \eta\right]
\end{aligned}
$$

for $0 \leq s \leq 1$. Firstly, in the light of the given condition $(\mathbb{C} 1)$, it is definite that for each $0 \leq s \leq 1$,

$$
\left|\hbar_{2}(s, \omega(s))\right| \leq\left[\left|\hbar_{2}(s, \omega(s))-\hbar_{2}(s, 0)\right|+\left|\hbar_{2}(s, 0)\right|\right] \leq \hat{L}^{*}|\omega(s)|+\hat{\mathbb{H}}_{*} .
$$

Further, the second condition $(\mathbb{C} 2)$ yields $\left|\hbar_{1}(s, \omega)\right| \leq \Phi(s)$ for $0 \leq s \leq 1$. In this case, for two arbitrary $\omega_{1}, \omega_{2} \in \mathbb{B}_{\tilde{r}}$, we may have 


$$
\begin{aligned}
& \left|\mathbb{T}_{1} \omega_{1}(s)+\mathbb{T}_{2} \omega_{2}(s)\right| \\
& \leq \frac{1}{K_{1}} \mathcal{R}_{\mathcal{I}_{0^{+}}^{\sigma}}\left(\hat{L}^{*}\|\omega\|+\hat{\mathbb{H}}_{*}\right)+\frac{K_{2}}{K_{1}} \int_{0}^{s} \frac{(s-\eta)^{\sigma+\theta-1}}{\Gamma(\sigma+\theta)}\|\omega\| \mathrm{d} \eta \\
& +\frac{s^{2}}{\xi K_{1}}\left[{ }^{\mathcal{R}} \mathcal{I}_{0^{+}}^{\sigma+q_{2}}\left(\hat{L}^{*}\|\omega\|+\hat{\mathbb{H}}_{*}\right)+\int_{0}^{1} \mathcal{R} \mathcal{I}_{0^{+}}^{\sigma}\left(\hat{L}^{*}\|\omega\|+\hat{\mathbb{H}}_{*}\right) \mathrm{d} \eta+\int_{0}^{1} \mathcal{R}_{\mathcal{I}_{0^{+}}^{\sigma-1}}\left(\hat{L}^{*}\|\omega\|+\hat{\mathbb{H}}_{*}\right) \mathrm{d} \eta\right] \\
& +\frac{s^{2} K_{2}}{\xi K_{1}}\left[\int_{0}^{1} \frac{(1-\eta)^{\sigma+\theta+q_{2}-1}}{\Gamma\left(\sigma+\theta+q_{2}\right)}\|\omega\| \mathrm{d} \eta+\int_{0}^{1} \int_{0}^{\eta} \frac{(\eta-r)^{\sigma+\theta-1}}{\Gamma(\sigma+\theta)}\|\omega\| \mathrm{d} r \mathrm{~d} \eta\right. \\
& \left.+\int_{0}^{1} \int_{0}^{\eta} \frac{(\eta-r)^{\sigma+\theta-2}}{\Gamma(\sigma+\theta-1)}\|\omega\| \mathrm{d} r \mathrm{~d} \eta\right]+\frac{1}{K_{1}} \mathcal{R}_{\mathcal{I}^{+}}^{\sigma+q_{1}}\|\Phi\| \\
& +\frac{s^{2}}{\xi K_{1}}\left[{ }^{\mathcal{R}} \mathcal{I}_{0^{+}}^{\sigma+q_{2}+q_{1}}\|\Phi\|+\int_{0}^{1} \mathcal{R}_{\mathcal{I}_{0^{+}}^{\sigma+q_{1}}}\|\Phi\| \mathrm{d} \eta+\int_{0}^{1}{ }^{\mathcal{R}} \mathcal{I}_{0^{+}}^{\sigma+q_{1}-1}\|\Phi\| \mathrm{d} \eta\right] \\
& \leq \frac{1}{K_{1} \Gamma(\sigma+1)}\left(\hat{L}^{*}\|\omega\|+\hat{\mathbb{H}}_{*}\right)+\frac{K_{2}}{K_{1}} \frac{(s)^{\sigma+\theta}}{\Gamma(\sigma+\theta+1)}\|\omega\| \\
& +\frac{1}{\xi K_{1}}\left[\frac{1}{\Gamma\left(\sigma+q_{2}+1\right)}\left(\hat{L}^{*}\|\omega\|+\hat{\mathbb{H}}_{*}\right)+\frac{1}{\Gamma(\sigma+2)}\left(\hat{L}^{*}\|\omega\|+\hat{\mathbb{H}}_{*}\right)+\frac{1}{\Gamma(\sigma+1)}\left(\hat{L}^{*}\|\omega\|+\hat{\mathbb{H}}_{*}\right)\right] \\
& +\frac{K_{2}}{\xi K_{1}}\left[\frac{1}{\Gamma\left(\sigma+\theta+q_{2}+1\right)}\|\omega\|+\frac{1}{\Gamma(\sigma+\theta+2)}\|\omega\|+\frac{1}{\Gamma(\sigma+\theta+1)}\|\omega\|\right]+\frac{1}{K_{1} \Gamma\left(\sigma+q_{1}+1\right)}\|\Phi\| \\
& +\frac{1}{\xi K_{1}}\left[\frac{1}{\Gamma\left(\sigma+q_{2}+q_{1}+1\right)}\|\Phi\|+\frac{1}{\Gamma\left(\sigma+q_{1}+2\right)}\|\Phi\|+\frac{1}{\Gamma\left(\sigma+q_{1}+1\right)}\|\Phi\|\right] \\
& =\left(\tilde{\Delta}^{(1)}+\hat{L}^{*} \tilde{\Delta}^{(2)}\right)\left\|\omega_{1}\right\|+\tilde{\Delta}^{(3)}\|\Phi\|+\tilde{\Delta}_{*}^{(2)} \hat{\mathbb{H}}_{*} \\
& \leq\left(\tilde{\Delta}^{(1)}+\hat{L}^{*} \tilde{\Delta}^{(2)}\right) \tilde{r}+\tilde{\Delta}^{(3)}\|\Phi\|+\tilde{\Delta}^{(2)} \hat{\mathbb{H}}_{*} \leq \tilde{r} .
\end{aligned}
$$

The above obtained inequality certifies that $\left\|\mathbb{T}_{1} \omega_{1}+\mathbb{T}_{2} \omega_{2}\right\| \leq \tilde{r}$ and accordingly $\mathbb{T}_{1} \omega_{1}+\mathbb{T}_{2} \omega_{2} \in \mathbb{B}_{\tilde{r}}$ for all $\omega_{1}, \omega_{2} \in \mathbb{B}_{\tilde{r}}$. The mentioned result also means that the hypothesis (th1) in Theorem 1 is valid about $\mathbb{T}_{1}$ and $\mathbb{T}_{2}$. In the next stage, we confirm the contractivity of $\mathbb{T}_{1}$. For two arbitrary $\omega_{1}, \omega_{2} \in \mathbb{B}_{\tilde{r}}$ and $0 \leq s \leq 1$ and in the light of $(\mathbb{C} 1)$, we can write 


$$
\begin{aligned}
& \left|\mathbb{T}_{1} \omega_{1}(s)-\mathbb{T}_{1} \omega_{2}(s)\right| \\
\leq & \frac{1}{K_{1}} \mathcal{R}_{\mathcal{I}^{+}}^{\sigma} \hat{L}^{*}\left|\omega_{1}(s)-\omega_{2}(s)\right|+\frac{K_{2}}{K_{1}} \int_{0}^{s} \frac{(s-\eta)^{\sigma+\theta-1}}{\Gamma(\sigma+\theta)}\left|\omega_{1}(\eta)-\omega_{2}(\eta)\right| \mathrm{d} \eta \\
& +\frac{s^{2}}{\xi K_{1}}\left[{ }^{\mathcal{R}} \mathcal{I}_{0^{+}}^{\sigma+q_{2}} \hat{L}^{*}\left|\omega_{1}-\omega_{2}\right|+\int_{0}^{1}{ }^{\mathcal{R}} \mathcal{I}_{0^{+}}^{\sigma} \hat{L}^{*}\left|\omega_{1}(\eta)-\omega_{2}(\eta)\right| \mathrm{d} \eta\right. \\
& +\int_{0}^{1} \mathcal{R}_{\left.\mathcal{I}_{0^{+}}^{\sigma-1} \hat{L}^{*}\left|\omega_{1}(\eta)-\omega_{2}(\eta)\right| \mathrm{d} \eta\right]} \\
& +\frac{s^{2} K_{2}}{\xi^{\xi} K_{1}}\left[\int_{0}^{1} \frac{(1-\eta)^{\sigma+\theta+q_{2}-1}}{\Gamma\left(\sigma+\theta+q_{2}\right)}\left|\omega_{1}(\eta)-\omega_{2}(\eta)\right| \mathrm{d} \eta+\int_{0}^{1} \int_{0}^{\eta} \frac{(\eta-r)^{\sigma+\theta-1}}{\Gamma(\sigma+\theta)}\left|\omega_{1}(r)-\omega_{2}(r)\right| \mathrm{d} r \mathrm{~d} \eta\right. \\
& \left.+\int_{0}^{1} \int_{0}^{\eta} \frac{(\eta-r)^{\sigma+\theta-2}}{\Gamma(\sigma+\theta-1)}\left|\omega_{1}(r)-\omega_{2}(r)\right| \mathrm{d} r \mathrm{~d} \eta\right] \\
= & \left(\tilde{\Delta}^{(1)}+\hat{L}^{*} \tilde{\Delta}^{(2)}\right)\left\|\omega_{1}-\omega_{2}\right\| .
\end{aligned}
$$

By virtue of the hypothesis, it is apparent that $\tilde{\Delta}^{(1)}+\hat{L}^{*} \tilde{\Delta}^{(2)}<1$. Therefore we find that $\mathbb{T}_{1}$ possesses the important property of contractivity and thus the second condition (th2) in Theorem 1 holds about $\mathbb{T}_{1}$.

Next, we focus on the continuity of $\mathbb{T}_{2}$. Toward this purpose, we regard $\left\{\omega_{n}\right\}_{n \geq 1}$ as a sequence contained in the defined ball $\mathbb{B}_{\tilde{r}}$ so that $\omega_{n}$ goes to $\omega$. In this case, for $0 \leq s \leq 1$, we get that

$$
\begin{aligned}
& \left|\mathbb{T}_{2} \omega_{n}(s)-\mathbb{T}_{2} \omega(s)\right| \\
\leq & \frac{K_{2}}{K_{1} \Gamma(\sigma+\theta+1)}\left|\hbar_{1}\left(s, \omega_{n}(s)\right)-\hbar_{1}(s, \omega(s))\right| \\
& +\frac{K_{2}}{\xi K_{1}}\left[\frac{1}{\Gamma\left(\sigma+\theta+q_{2}+1\right)}\left|\hbar_{1}\left(s, \omega_{n}(s)\right)-\hbar_{1}(s, \omega(s))\right|\right. \\
& \left.+\frac{1}{\Gamma(\sigma+\theta+2)}\left|\hbar_{1}\left(s, \omega_{n}(s)\right)-\hbar_{1}(s, \omega(s))\right|+\frac{1}{\Gamma(\sigma+\theta+1)}\left|\hbar_{1}\left(s, \omega_{n}(s)\right)-\hbar_{1}(s, \omega(s))\right|\right] .
\end{aligned}
$$

On the other side, note that the map $\hbar_{1}$ is continuous on $[0,1] \times X$. Hence we observe that $\left\|\mathbb{T}_{2} \omega_{n}-\mathbb{T}_{2} \omega\right\|$ goes to 0 when $\omega_{n} \rightarrow \omega$. Consequently, we perceive that $\mathbb{T}_{2}$ is continuous on $\mathbb{B}_{\tilde{r}}$. Next, we survey the compactness of $\mathbb{T}_{2}$. To accomplish this purpose, we first have to survey the uniform boundedness of $\mathbb{T}_{2}$. For $\omega \in \mathbb{B}_{\tilde{r}}$ and $0 \leq s \leq 1$, we estimate 
$\left|\mathbb{T}_{2} \omega(s)\right|$

$$
\begin{aligned}
\leq & \frac{1}{K_{1}}{ }^{\mathcal{R}} \mathcal{I}_{0^{+}}^{\sigma+q_{1}}\left|\hbar_{1}(s, \omega(s))\right|+\frac{s^{2}}{\xi K_{1}}\left[\mathcal{R}_{\mathcal{I}_{0^{+}}^{\sigma+q_{2}+q_{1}}\left|\hbar_{1}(1, \omega(1))\right|+\int_{0}^{1} \mathcal{R}^{\mathcal{I}} \mathcal{I}_{0^{+}}^{\sigma+q_{1}}\left|\hbar_{1}(\eta, \omega(\eta))\right| \mathrm{d} \eta}\right. \\
& \left.+\int_{0}^{1}{ }^{\mathcal{R}} \mathcal{I}_{0^{+}}^{\sigma+q_{1}-1}\left|\hbar_{1}(\eta, \omega(\eta))\right| \mathrm{d} \eta\right] \\
\leq & \|\Phi\|\left[\frac{1}{K_{1} \Gamma\left(\sigma+q_{1}+1\right)}+\frac{1}{\xi K_{1}}\left[\frac{1}{\Gamma\left(\sigma+q_{2}+q_{1}+1\right)}+\frac{1}{\Gamma\left(\sigma+q_{1}+2\right)}+\frac{1}{\Gamma\left(\sigma+q_{1}+1\right)}\right]\right] \\
= & \tilde{\Delta}^{(3)}\|\Phi\|,
\end{aligned}
$$

which displays that $\left\|\mathbb{T}_{2} \omega\right\| \leq \tilde{\Delta}^{(3)}\|\Phi\|$ and ergo $\mathbb{T}_{2}$ includes the property of the uniform boundedness. Furthermore, we assert that $\mathbb{T}_{2}$ is equi-continuous. To reach this goal, we select two arbitrary variables $0 \leq s, x \leq 1$ subject to $s<x$. In fact, we assert that bounded sets are mapped to equi-continuous sets by $\mathbb{T}_{2}$. Thus, for any $\omega \in \mathbb{B}_{\tilde{r}}$, we reach

$$
\begin{aligned}
& \left|\mathbb{T}_{2} \omega(x)-\mathbb{T}_{2} \omega(s)\right| \\
& \leq \frac{1}{K_{1}}\left[\int_{0}^{s}\left(\frac{(x-r)^{\sigma+q_{1}-1}-(s-r)^{\sigma+q_{1}-1}}{\Gamma\left(\sigma+q_{1}\right)}\right) \hbar_{1}(r, \omega(r)) \mathrm{d} r+\int_{s}^{x} \frac{(x-r)^{\sigma+q_{1}-1}}{\Gamma\left(\sigma+q_{1}\right)} \hbar_{1}(r, \omega(r)) \mathrm{d} r\right] \\
& +\frac{\left(x^{2}-s^{2}\right)}{\xi K_{1}}\left[\mathcal{R}^{\mathcal{I}} \mathcal{I}^{\sigma+q_{2}+q_{1}} \hbar_{1}(1, \omega(1))+\int_{0}^{1}{ }^{\mathcal{R}} \mathcal{I}_{0^{+}}^{\sigma+q_{1}} \hbar_{1}(\eta, \omega(\eta)) \mathrm{d} \eta+\int_{0}^{1}{ }^{\mathcal{R}} \mathcal{I}_{0^{+}}^{\sigma+q_{1}-1} \hbar_{1}(\eta, \omega(\eta)) \mathrm{d} \eta\right] \\
& \leq \frac{\|\Phi\|}{K_{1}}\left[\int_{0}^{s}\left(\frac{(x-r)^{\sigma+q_{1}-1}-(s-r)^{\sigma+q_{1}-1}}{\Gamma\left(\sigma+q_{1}\right)}\right) \mathrm{d} r+\int_{s}^{x} \frac{(x-r)^{\sigma+q_{1}-1}}{\Gamma\left(\sigma+q_{1}\right)} \mathrm{d} r\right] \\
& +\frac{\left(x^{2}-s^{2}\right)\|\Phi\|}{\xi K_{1}}\left[{ }^{\mathcal{R}} \mathcal{I}_{0^{+}}^{\sigma+q_{2}+q_{1}}(1)+\int_{0}^{1}{ }^{\mathcal{R}} \mathcal{I}_{0^{+}}^{\sigma+q_{1}}(1) \mathrm{d} \eta+\int_{0}^{1}{ }^{\mathcal{R}} \mathcal{I}_{0^{+}}^{\sigma+q_{1}-1}(1) \mathrm{d} \eta\right] .
\end{aligned}
$$

The R.H.S of the above is not dependent on $\omega \in \mathbb{B}_{\tilde{r}}$ and goes to zero by tending $s$ to $x$. As a result, we find that $\mathbb{T}_{2}$ is equi-continuous. Finally, it is perceived that $\mathbb{T}_{2}$ involves the property of relative compactness on $\omega \in \mathbb{B}_{\tilde{r}}$ and thus the Arzelà-Ascoli result expresses that $\mathbb{T}_{2}$ is completely continuous and finally is compact on the defined ball $\omega \in \mathbb{B}_{\tilde{r}}$. Accordingly, the condition (th3) in Theorem 1 is met regarding the operator $\mathbb{T}_{2}$. Thereupon, all information for Theorem 1 is established for two existing single-valued maps $\mathbb{T}_{1}$ and $\mathbb{T}_{2}$. Consequently, Theorem 1 guarantees that the formulated nonlinear integro-differential CFBVP (1) involves a solution on $[0,1]$ and the argument is ended.

The Leray-Schauder nonlinear alternative result is a further deductive tool for which we are able to extract desired criteria of existence for the aforementioned nonlinear integrodifferential CFBVP (1).

Theorem 6. Regard $\hbar_{j}:[0,1] \times X \rightarrow X(j=1,2)$ as two continuous maps equipped with the following clauses:

$(\mathbb{C} 3) \mathrm{Y}_{1}, \mathrm{Y}_{2} \in \mathcal{C}\left([0,1], \mathbb{R}^{+}\right)$along with two continuous nondecreasing maps $\mathfrak{N}_{1}, \mathfrak{N}_{2}:[0, \infty) \rightarrow$ $(0, \infty)$ exist provided for all $(s, \omega) \in[0,1] \times X$,

$$
\left|\hbar_{2}(s, \omega)\right| \leq \mathrm{Y}_{1}(s) \mathfrak{N}_{1}(|\omega|) \text { and }\left|\hbar_{1}(s, \omega)\right| \leq \mathrm{Y}_{2}(s) \mathfrak{N}_{2}(|\omega|) ;
$$


(C4) A number $\varepsilon>0$ exists provided that $\tilde{\Delta}^{(1)}<1$ and

$$
\frac{\left(1-\tilde{\Delta}^{(1)}\right) \varepsilon}{\tilde{\Delta}^{(2)}\left\|\mathrm{Y}_{1}\right\| \mathfrak{N}_{1}(\varepsilon)+\tilde{\Delta}^{(3)}\left\|\mathrm{Y}_{2}\right\| \mathfrak{N}_{2}(\varepsilon)}>1,
$$

where $\tilde{\Delta}^{(k)}(k=1,2,3)$ are displayed in (8).

Then the nonlinear integro-differential CFBVP (1) involves a solution on $[0,1]$.

Proof. To arrive at required result, we establish all information of the Leray-Schauder nonlinear alternative (Theorem 2) in the steps ahead. First, we check that the operator $\mathbb{T}$ exhibited as (7) corresponds bounded sets (i.e., balls) into ones contained in $X$. For $\tilde{R} \in \mathbb{R}^{+}$, a bounded ball $\mathbb{B}_{\tilde{R}}=\{\omega \in X:\|\omega\| \leq \tilde{R}\}$ is constructed in $X$. In this phase, for any $0 \leq s \leq 1$ and in the light of $(\mathbb{C} 3)$, we get that

$|\mathbb{T} \omega(s)|$

$$
\begin{aligned}
& \leq \frac{1}{K_{1}} \mathcal{R}_{0^{+}}^{\sigma+q_{1}}\left\|\mathrm{Y}_{2}\right\| \mathfrak{N}_{2}(\|\omega\|)+\frac{1}{K_{1}} \mathcal{R}_{\mathcal{I}^{+}}^{\sigma}\left\|\mathrm{Y}_{1}\right\| \mathfrak{N}_{1}(\|\omega\|)+\frac{K_{2}}{K_{1}} \int_{0}^{s} \frac{(s-\eta)^{\sigma+\theta-1}}{\Gamma(\sigma+\theta)}\|\omega\| \mathrm{d} \eta
\end{aligned}
$$

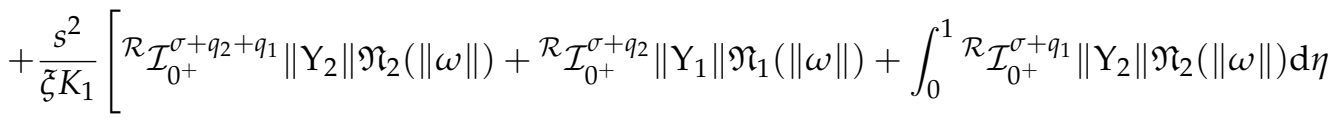

$$
\begin{aligned}
& \left.+\int_{0}^{1} \mathcal{R}_{\mathcal{I}_{0^{+}}^{\sigma}}\left\|\mathrm{Y}_{1}\right\| \mathfrak{N}_{1}(\|\omega\|) \mathrm{d} \eta-\int_{0}^{1}{ }^{\mathcal{R}} \mathcal{I}_{0^{+}}^{\sigma+q_{1}-1}\left\|\mathrm{Y}_{2}\right\| \mathfrak{N}_{2}(\|\omega\|) \mathrm{d} \eta+\int_{0}^{1}{ }^{\mathcal{R}} \mathcal{I}_{0^{+}}^{\sigma-1}\left\|\mathrm{Y}_{1}\right\| \mathfrak{N}_{1}(\|\omega\|) \mathrm{d} \eta\right] \\
& +\frac{s^{2} K_{2}}{\xi K_{1}}\left[\int_{0}^{1} \frac{(1-\eta)^{\sigma+\theta+q_{2}-1}}{\Gamma\left(\sigma+\theta+q_{2}\right)}\|\omega\| \mathrm{d} \eta+\int_{0}^{1} \int_{0}^{\eta} \frac{(\eta-r)^{\sigma+\theta-1}}{\Gamma(\sigma+\theta)}\|\omega\| \mathrm{d} r \mathrm{~d} \eta\right. \\
& \left.+\int_{0}^{1} \int_{0}^{\eta} \frac{(\eta-r)^{\sigma+\theta-2}}{\Gamma(\sigma+\theta-1)}\|\omega\| \mathrm{d} r \mathrm{~d} \eta\right] \\
& =\tilde{\Delta}^{(1)}\|\omega\|+\tilde{\Delta}^{(2)}\left\|\mathrm{Y}_{1}\right\| \mathfrak{N}_{1}(\|\omega\|)+\tilde{\Delta}^{(3)}\left\|\mathrm{Y}_{2}\right\| \mathfrak{N}_{2}(\|\omega\|) .
\end{aligned}
$$

Thus, from the above relations, we comprehend that

$$
\|\mathbb{T} \omega\| \leq \tilde{\Delta}^{(1)} \tilde{R}+\tilde{\Delta}^{(2)}\left\|\mathrm{Y}_{1}\right\| \mathfrak{N}_{1}(\tilde{R})+\tilde{\Delta}^{(3)}\left\|\mathrm{Y}_{2}\right\| \mathfrak{N}_{2}(\tilde{R}) .
$$

The obtained inequality states that the operator $\mathbb{T}$ involves the uniform boundedness specification. In the further stage, we implement an argument for verifying this issue where $\mathbb{T}$ maps bounded subsets (i.e., balls) into equi-continuous ones in $X$. To view this goal, select $s, x \in[0,1]$ arbitrarily subject to $s<x$ and $\omega \in \mathbb{B}_{\tilde{R}}$. In that case, we can estimate 


$$
\begin{aligned}
& |\mathbb{T} \omega(x)-\mathbb{T} \omega(s)| \\
& \leq \frac{\left\|\mathrm{Y}_{2}\right\| \mathfrak{N}_{2}(\|\omega\|)}{K_{1} \Gamma\left(\sigma+q_{1}\right)}\left[\int_{0}^{s}\left[(x-r)^{\sigma+q_{1}-1}-(s-r)^{\sigma+q_{1}-1}\right] \mathrm{d} r+\int_{s}^{x}(x-r)^{\sigma+q_{1}-1} \mathrm{~d} r\right] \\
& +\frac{\left\|\mathrm{Y}_{1}\right\| \mathfrak{N}_{1}(\|\omega\|)}{K_{1} \Gamma(\sigma)}\left[\int_{0}^{s}\left[(x-r)^{\sigma-1}-(s-r)^{\sigma-1}\right] \mathrm{d} r+\int_{s}^{x}(x-r)^{\sigma-1} \mathrm{~d} r\right] \\
& +\frac{K_{2} \tilde{R}}{K_{1} \Gamma(\sigma+\theta)}\left[\int_{0}^{s}\left[(x-r)^{\sigma+\theta-1}-(s-r)^{\sigma+\theta-1}\right] \mathrm{d} r+\int_{s}^{x}(x-r)^{\sigma+\theta-1} \mathrm{~d} r\right] \\
& +\frac{x^{2}-s^{2}}{\xi K_{1}}\left[{ }^{\mathcal{R}} \mathcal{I}_{0^{+}}^{\sigma+q_{2}+q_{1}}\left\|\mathrm{Y}_{2}\right\| \mathfrak{N}_{2}(\|\omega\|)+{ }^{\mathcal{R}} \mathcal{I}_{0^{+}}^{\sigma+q_{2}}\left\|\mathrm{Y}_{1}\right\| \mathfrak{N}_{1}(\|\omega\|)+\int_{0}^{1} \mathcal{R}^{\mathcal{I}_{0^{+}}^{\sigma+q_{1}}}\left\|\mathrm{Y}_{2}\right\| \mathfrak{N}_{2}(\|\omega\|) \mathrm{d} \eta\right. \\
& \left.+\int_{0}^{1} \mathcal{R}_{\mathcal{I}_{0^{+}}^{\sigma}}\left\|\mathrm{Y}_{1}\right\| \mathfrak{N}_{1}(\|\omega\|) \mathrm{d} \eta+\int_{0}^{1}{ }^{\mathcal{R}} \mathcal{I}_{0^{+}}^{\sigma+q_{1}-1}\left\|\mathrm{Y}_{2}\right\| \mathfrak{N}_{2}(\|\omega\|) \mathrm{d} \eta+\int_{0}^{1}{ }^{\mathcal{R}} \mathcal{I}_{0^{+}}^{\sigma-1}\left\|\mathrm{Y}_{1}\right\| \mathfrak{N}_{1}(\|\omega\|) \mathrm{d} \eta\right] \\
& +\frac{\left(x^{2}-s^{2}\right) K_{2}}{\xi K_{1}}\left[\int_{0}^{1} \frac{(1-\eta)^{\sigma+\theta+q_{2}-1}}{\Gamma\left(\sigma+\theta+q_{2}\right)} \tilde{R} \mathrm{~d} \eta+\int_{0}^{1} \int_{0}^{\eta} \frac{(\eta-r)^{\sigma+\theta-1}}{\Gamma(\sigma+\theta)} \tilde{R} \mathrm{~d} r \mathrm{~d} \eta\right. \\
& \left.+\int_{0}^{1} \int_{0}^{\eta} \frac{(\eta-r)^{\sigma+\theta-2}}{\Gamma(\sigma+\theta-1)} \tilde{R} \mathrm{~d} r \mathrm{~d} \eta\right] \\
& \leq \frac{\left\|\mathrm{Y}_{2}\right\| \mathfrak{N}_{2}(\tilde{R})}{K_{1} \Gamma\left(\sigma+q_{1}\right)}\left[\int_{0}^{s}\left[(x-r)^{\sigma+q_{1}-1}-(s-r)^{\sigma+q_{1}-1}\right] \mathrm{d} r+\int_{s}^{x}(x-r)^{\sigma+q_{1}-1} \mathrm{~d} r\right] \\
& +\frac{\left\|\mathrm{Y}_{1}\right\| \mathfrak{N}_{1}(\tilde{R})}{K_{1} \Gamma(\sigma)}\left[\int_{0}^{s}\left[(x-r)^{\sigma-1}-(s-r)^{\sigma-1}\right] \mathrm{d} r+\int_{s}^{x}(x-r)^{\sigma-1} \mathrm{~d} r\right] \\
& +\frac{K_{2} \tilde{R}}{K_{1} \Gamma(\sigma+\theta)}\left[\int_{0}^{s}\left[(x-r)^{\sigma+\theta-1}-(s-r)^{\sigma+\theta-1}\right] \mathrm{d} r+\int_{s}^{x}(x-r)^{\sigma+\theta-1} \mathrm{~d} r\right] \\
& +\frac{x^{2}-s^{2}}{\xi K_{1}}\left[\mathcal{R}_{\mathcal{I}^{+}}^{\sigma+q_{2}+q_{1}}\left\|\mathrm{Y}_{2}\right\| \mathfrak{N}_{2}(\tilde{R})+{ }^{\mathcal{R}} \mathcal{I}_{0^{+}}^{\sigma+q_{2}}\left\|\mathrm{Y}_{1}\right\| \mathfrak{N}_{1}(\tilde{R})+\int_{0}^{1}{ }^{1} \mathcal{I}_{0^{+}}^{\sigma+q_{1}}\left\|\mathrm{Y}_{2}\right\| \mathfrak{N}_{2}(\tilde{R}) \mathrm{d} \eta\right. \\
& \left.+\int_{0}^{1} \mathcal{R}_{\mathcal{I}_{0^{+}}^{\sigma}}\left\|\mathrm{Y}_{1}\right\| \mathfrak{N}_{1}(\tilde{R}) \mathrm{d} \eta+\int_{0}^{1}{ }^{\mathcal{R}} \mathcal{I}_{0^{+}}^{\sigma+q_{1}-1}\left\|\mathrm{Y}_{2}\right\| \mathfrak{N}_{2}(\tilde{R}) \mathrm{d} \eta+\int_{0}^{1} \mathcal{R}_{\mathcal{I}_{0^{+}}^{\sigma-1}}\left\|\mathrm{Y}_{1}\right\| \mathfrak{N}_{1}(\tilde{R}) \mathrm{d} \eta\right] \\
& +\frac{\left(x^{2}-s^{2}\right) K_{2}}{\xi K_{1}}\left[\int_{0}^{1} \frac{(1-\eta)^{\sigma+\theta+q_{2}-1}}{\Gamma\left(\sigma+\theta+q_{2}\right)} \tilde{R} \mathrm{~d} \eta+\int_{0}^{1} \int_{0}^{\eta} \frac{(\eta-r)^{\sigma+\theta-1}}{\Gamma(\sigma+\theta)} \tilde{R} \mathrm{~d} r \mathrm{~d} \eta\right. \\
& \left.+\int_{0}^{1} \int_{0}^{\eta} \frac{(\eta-r)^{\sigma+\theta-2}}{\Gamma(\sigma+\theta-1)} \tilde{R} \mathrm{~d} r \mathrm{~d} \eta\right] .
\end{aligned}
$$

We discover that the R.H.S of the above is not dependent on $\omega \in \mathbb{B}_{\tilde{R}}$ and also goes to zero by tending $s$ to $x$. As a result, $\mathbb{T}$ is equi-continuous and ergo the complete continuity of $\mathbb{T}: X \rightarrow X$ is validated with the help of the Arzelà-Ascoli result. Consequently, $\mathbb{G}$ is compact. 
Finally, for the sake of holding all clauses of the Leray-Schauder nonlinear result (Theorem 2), we corroborate that the collection of all existing solutions of an equation $\omega=\hat{a}(\mathbb{T} \omega)$ is bounded for $\hat{a} \in[0,1]$. To view this, we regard $\omega^{*}$ as a solution for the equation $\omega^{*}=\hat{a} \mathbb{T} \omega^{*}$ for $\hat{a} \in[0,1]$. In that case, by employing the argument used in the first stage for all $s \in[0,1]$, we reach

$$
\left\|\omega^{*}\right\| \leq \tilde{\Delta}^{(1)}\left\|\omega^{*}\right\|+\tilde{\Delta}^{(2)}\left\|\mathrm{Y}_{1}\right\| \mathfrak{N}_{1}\left(\left\|\omega^{*}\right\|\right)+\tilde{\Delta}^{(3)}\left\|\mathrm{Y}_{2}\right\| \mathfrak{N}_{2}\left(\left\|\omega^{*}\right\|\right) .
$$

In this phase, we have

$$
\frac{\left(1-\tilde{\Delta}^{(1)}\right)\left\|\omega^{*}\right\|}{\tilde{\Delta}^{(2)}\left\|\mathrm{Y}_{1}\right\| \mathfrak{N}_{1}\left(\left\|\omega^{*}\right\|\right)+\tilde{\Delta}^{(3)}\left\|\mathrm{Y}_{2}\right\| \mathfrak{N}_{2}\left(\left\|\omega^{*}\right\|\right)} \leq 1
$$

With regard to the hypothesis $(\mathbb{C} 4)$, one can pick up a real number $\varepsilon>0$ provided $\left\|\omega^{*}\right\| \neq \varepsilon$. On the other side, we introduce

$$
\mathbb{W}=\left\{\omega^{*} \in X:\left\|\omega^{*}\right\|<\varepsilon\right\} .
$$

It is easy to see that $\mathbb{T}: \overline{\mathbb{W}} \rightarrow X$ possesses the properties of continuity and complete continuity. With regard to this selection of $\mathbb{W}$, we cannot find $\omega^{*} \in \partial \mathbb{W}$ which satisfies $\omega^{*}=\hat{a}\left(\mathbb{T} \omega^{*}\right)$ for some $\hat{a} \in(0,1)$. Finally, by Theorem 2 , we conclude that the operator $\mathbb{T}$ contains a fixed point attached to the ball $\mathbb{W}$. Accordingly, there exists a solution leastwise on $[0,1]$ for the nonlinear integro-differential CFBVP (1) and so our proof is ended.

This portion of the current section aims to concentrate on the criterion of the uniqueness for possible solutions of the aforesaid nonlinear integro-differential CFBVP (1) with regard to the Banach principle (Theorem 3).

Theorem 7. Suppose the function $\hbar_{2}:[0,1] \times X \rightarrow X$ satisfies $(\mathbb{C} 1)$ and the following holds for $\hbar_{1}:[0,1] \times X \rightarrow X$ as:

(C) A number $\mathfrak{R} \in \mathbb{R}^{+}$exists so that for all $\omega_{1}, \omega_{2} \in X$, we have

$$
\left|\hbar_{1}\left(s, \omega_{1}\right)-\hbar_{1}\left(s, \omega_{2}\right)\right| \leq \mathfrak{R}\left|\omega_{1}-\omega_{2}\right|, \quad(s \in[0,1]) .
$$

In that case, a solution exists on $[0,1]$ uniquely for the nonlinear integro-differential CFBVP (1) provided that $\tilde{\Delta}^{(1)}+\hat{L}^{*} \tilde{\Delta}^{(2)}+\mathfrak{R} \tilde{\Delta}^{(3)}<1$, where $\tilde{\Delta}^{(j)}(j=1,2,3)$ are displayed in (8).

Proof. By invoking Theorem 3, we confirm that $\mathbb{T}: X \rightarrow X$ displayed by (7) possesses a fixed point uniquely which is equivalent to the existence of a solution unique for the aforementioned nonlinear integro-differential CFBVP (1). By adopting $\sup _{s \in[0,1]}\left|\hbar_{2}(s, 0)\right|=$ $\hat{\mathbb{H}}_{*}<\infty$ and $\sup _{s \in[0,1]}\left|\hbar_{1}(s, 0)\right|=\hat{\mathbb{G}}_{*}<\infty$ and picking up $\tilde{d}>0$ provided

$$
\tilde{d} \geq \frac{\hat{\mathbb{G}}_{*} \tilde{\Delta}^{(3)}+\hat{\mathbb{H}}_{*} \tilde{\Delta}^{(2)}}{1-\left(\tilde{\Delta}^{(1)}+\hat{L}^{*} \tilde{\Delta}^{(2)}+\mathfrak{R} \tilde{\Delta}^{(3)}\right)}
$$

along with a bounded ball $\mathbb{B}_{\tilde{d}}=\{\omega \in X:\|\omega\| \leq \tilde{d}\}$, we assert that $\mathbb{T}\left(\mathbb{B}_{\tilde{d}}\right) \subset \mathbb{B}_{\tilde{d}}$. By choosing $\omega \in \mathbb{B}_{\tilde{d}}$ arbitrarily and with the help of $(\mathbb{C} 1)$ and $(\mathbb{C} 5)$, we have 
$\|\mathbb{T} \omega\|$

$$
\begin{aligned}
& \leq \frac{1}{K_{1}} \mathcal{R}_{\mathcal{I}^{+}}^{\sigma}\left(\hat{L}^{*}\|\omega\|+\hat{\mathbb{H}}_{*}\right)+\frac{K_{2}}{K_{1}} \int_{0}^{s} \frac{(s-\eta)^{\sigma+\theta-1}}{\Gamma(\sigma+\theta)}\|\omega\| \mathrm{d} \eta \\
& +\frac{s^{2}}{\xi K_{1}}\left[{ }^{\mathcal{R}} \mathcal{I}_{0^{+}}^{\sigma+q_{2}}\left(\hat{L}^{*}\|\omega\|+\hat{\mathbb{H}}_{*}\right)+\int_{0}^{1}{ }^{\mathcal{R}} \mathcal{I}_{0^{+}}^{\sigma}\left(\hat{L}^{*}\|\omega\|+\hat{\mathbb{H}}_{*}\right) \mathrm{d} \eta+\int_{0}^{1}{ }^{\mathcal{R}} \mathcal{I}_{0^{+}}^{\sigma-1}\left(\hat{L}^{*}\|\omega\|+\hat{\mathbb{H}}_{*}\right) \mathrm{d} \eta\right] \\
& +\frac{s^{2} K_{2}}{\xi K_{1}}\left[\int_{0}^{1} \frac{(1-\eta)^{\sigma+\theta+q_{2}-1}}{\Gamma\left(\sigma+\theta+q_{2}\right)}\|\omega\| \mathrm{d} \eta+\int_{0}^{1} \int_{0}^{\eta} \frac{(\eta-r)^{\sigma+\theta-1}}{\Gamma(\sigma+\theta)}\|\omega\| \mathrm{d} r \mathrm{~d} \eta\right. \\
& \left.+\int_{0}^{1} \int_{0}^{\eta} \frac{(\eta-r)^{\sigma+\theta-2}}{\Gamma(\sigma+\theta-1)}\|\omega\| \mathrm{d} r \mathrm{~d} \eta\right]+\frac{1}{K_{1}} \mathcal{R}_{0^{+}}^{\sigma+q_{1}}\left(\mathfrak{R}\|\omega\|+\hat{\mathbb{G}}_{*}\right) \\
& +\frac{s^{2}}{\xi K_{1}}\left[\mathcal{R}^{\mathcal{I}} \mathcal{I}_{0^{+}}^{\sigma+q_{2}+q_{1}}\left(\mathfrak{R}\|\omega\|+\hat{\mathbb{G}}_{*}\right)+\int_{0}^{1} \mathcal{R}_{\mathcal{I}_{0^{+}}}^{\sigma+q_{1}}\left(\mathfrak{R}\|\omega\|+\hat{\mathbb{G}}_{*}\right) \mathrm{d} \eta\right.
\end{aligned}
$$

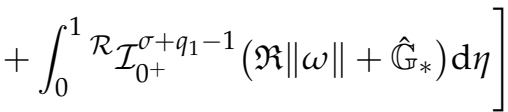

$$
\begin{aligned}
& \leq\left(\tilde{\Delta}^{(1)}+\hat{L}^{*} \tilde{\Delta}^{(2)}+\mathfrak{R} \tilde{\Delta}^{(3)}\right) \tilde{d}+\tilde{\Delta}^{(3)} \hat{\mathbb{G}}_{*}+\tilde{\Delta}^{(2)} \hat{\mathbb{H}}_{*}<\tilde{d} .
\end{aligned}
$$

In the light of the above outcome, it can be seen that the aforementioned assertion holds, and so we get that $\mathbb{T}\left(\mathbb{B}_{\tilde{d}}\right) \subset \mathbb{B}_{\tilde{d}}$. To validate the fact that $\mathbb{T}: X \rightarrow X$ introduced by (7) is a contraction, take that $0 \leq s \leq 1$ and $\omega_{1}, \omega_{2} \in X$ are arbitrary. In the current place, with regard to some direct calculations, we can simply comprehend that

$$
\left\|\left(\mathbb{T} \omega_{1}\right)-\left(\mathbb{T} \omega_{2}\right)\right\| \leq\left(\tilde{\Delta}^{(1)}+\hat{L}^{*} \tilde{\Delta}^{(2)}+\mathfrak{R} \tilde{\Delta}^{(3)}\right)\left\|\omega_{1}-\omega_{2}\right\| .
$$

In conclusion, the hypothesis $\tilde{\Delta}^{(1)}+\hat{L}^{*} \tilde{\Delta}^{(2)}+\mathfrak{R} \tilde{\Delta}^{(3)}<1$ indicates that $\mathbb{T}$ is a contraction. Therefore, the statement of Theorem 3 reveals that $\mathbb{T}$ involves a fixed point uniquely. Consequently, a solution exists uniquely for the nonlinear integro-differential CFBVP (1) and this completes our proof.

\section{Dependence of Solutions: A Special Case}

Here, we consider the dependence of solutions for a particular case of the given integro-differential CFBVP (1). To do this, we apply the generalized version of the Gronwall inequality. In precise terms, we take $K_{2}=0$ and $\hbar_{2} \equiv 0$. In this phase, we arrive at a new standard integro-differential CFBVP which takes the format

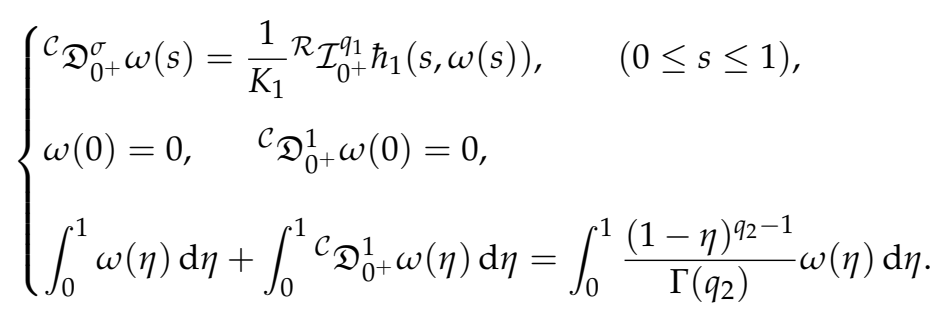

By invoking Proposition 1, it is easy to find that the solution of (9) takes the form 


$$
\begin{aligned}
\omega(s)= & \frac{1}{K_{1}} \int_{0}^{s} \frac{(s-\eta)^{\sigma+q_{1}-1}}{\Gamma\left(\sigma+q_{1}\right)} \hbar_{1}(\eta, \omega(\eta)) \mathrm{d} \eta \\
& +\frac{s^{2}}{\xi K_{1}}\left[\int_{0}^{1} \frac{(1-\eta)^{\sigma+q_{2}+q_{1}-1}}{\Gamma\left(\sigma+q_{2}+q_{1}\right)} \hbar_{1}(\eta, \omega(\eta)) \mathrm{d} \eta-\int_{0}^{1} \int_{0}^{\eta} \frac{(\eta-r)^{\sigma+q_{1}-1}}{\Gamma\left(\sigma+q_{1}\right)} \hbar_{1}(r, \omega(r)) \mathrm{d} r \mathrm{~d} \eta\right. \\
& \left.-\int_{0}^{1} \int_{0}^{\eta} \frac{(\eta-r)^{\sigma+q_{1}-2}}{\Gamma\left(\sigma+q_{1}-1\right)} \hbar_{1}(r, \omega(r)) \mathrm{d} r \mathrm{~d} \eta\right]
\end{aligned}
$$

in which it is assumed that

$$
\xi:=\left|\frac{4 \Gamma\left(q_{2}+3\right)-6}{3 \Gamma\left(q_{2}+3\right)}\right| \neq 0 .
$$

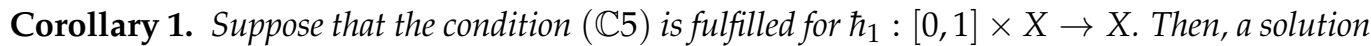
exists on $[0,1]$ uniquely for the nonlinear integro-differential CFBVP (9) subject to $\mathfrak{R} \tilde{\Delta}^{(3)}<1$, where $\tilde{\Delta}^{(3)}$ is displayed in (8).

Proof. With due attention to Theorem 3 , it is easy to check that $\mathbb{T}^{*}: X \rightarrow X$ illustrated by

$$
\begin{aligned}
\left(\mathbb{T}^{*} \omega\right)(s)= & \frac{1}{K_{1}} \int_{0}^{s} \frac{(s-\eta)^{\sigma+q_{1}-1}}{\Gamma\left(\sigma+q_{1}\right)} \hbar_{1}(\eta, \omega(\eta)) \mathrm{d} \eta \\
& +\frac{s^{2}}{\xi K_{1}}\left[\int_{0}^{1} \frac{(1-\eta)^{\sigma+q_{2}+q_{1}-1}}{\Gamma\left(\sigma+q_{2}+q_{1}\right)} \hbar_{1}(\eta, \omega(\eta)) \mathrm{d} \eta-\int_{0}^{1} \int_{0}^{\eta} \frac{(\eta-r)^{\sigma+q_{1}-1}}{\Gamma\left(\sigma+q_{1}\right)} \hbar_{1}(r, \omega(r)) \mathrm{d} r \mathrm{~d} \eta\right. \\
& \left.-\int_{0}^{1} \int_{0}^{\eta} \frac{(\eta-r)^{\sigma+q_{1}-2}}{\Gamma\left(\sigma+q_{1}-1\right)} \hbar_{1}(r, \omega(r)) \mathrm{d} r \mathrm{~d} \eta\right]
\end{aligned}
$$

admits one and exactly one fixed point which corresponds to a unique solution of (9). Adopt $\sup _{s \in[0,1]}\left|\hbar_{1}(s, 0)\right|=\hat{\mathbb{G}}_{*}<\infty$ and select $\tilde{d}_{*}>0$ so that

$$
\tilde{d}_{*} \geq \frac{\hat{\mathbb{G}}_{*} \tilde{\Delta}^{(3)}}{1-\mathfrak{R} \tilde{\Delta}^{(3)}}
$$

and build a bounded ball $\mathbb{B}_{\tilde{d}_{*}}=\left\{\omega \in X:\|\omega\| \leq \tilde{d}_{*}\right\}$. By a similar argument as in the proof of Theorem 7 , we get $\mathbb{T}^{*}\left(\mathbb{B}_{\tilde{d}_{*}}\right) \subset \mathbb{B}_{\tilde{d}_{*}}$, and for each $\omega_{1}, \omega_{2} \in X$, we obtain

$$
\left\|\left(\mathbb{T}^{*} \omega_{1}\right)-\left(\mathbb{T}^{*} \omega_{2}\right)\right\| \leq \Re \tilde{\Delta}^{(3)}\left\|\omega_{1}-\omega_{2}\right\|,
$$

which confirms that $\mathbb{T}^{*}$ is a contraction because $\mathfrak{R} \tilde{\Delta}^{(3)}<1$. Therefore, Theorem 3 states that $\mathbb{T}^{*}$ involves one and exactly one fixed point on its domain. So, a solution exists uniquely for the nonlinear integro-differential CFBVP (9).

Now, the dependence of solutions to the nonlinear integro-differential CFBVP (9) is studied by making a small change in the order $\sigma$. To reach this aim, we are helped by the generalized version of the Gronwall inequality. For more details, see [26,43].

Theorem 8. Let $\delta>0$ so that $2<\sigma-\delta<\sigma<3$. Let $\hbar_{1}:[0,1] \times X \rightarrow X$ be continuous and let there be $\ell>0$ subject to

$$
\left|\hbar_{1}(s, \omega(s))-\hbar_{1}\left(s, \omega^{\prime}(s)\right)\right| \leq \ell\left|\omega(s)-\omega^{\prime}(s)\right|
$$


for each $\omega, \omega^{\prime} \in X$ and $s \in[0,1]$. Further, assume $\omega$ and $\bar{\omega}$ are solutions of the nonlinear integro-differential CFBVP (9) and the integro-differential CFBVP

$$
\left\{\begin{array}{l}
\mathcal{C}_{\mathfrak{D}_{0^{+}}^{\sigma-\delta}} \bar{\omega}(s)=\frac{1}{K_{1}} \mathcal{R}_{\mathcal{I}_{0^{+}}}^{q_{1}} \hbar_{1}(s, \bar{\omega}(s)), \quad(0 \leq s \leq 1), \\
\bar{\omega}(0)=0, \quad \mathcal{C}_{\mathfrak{D}_{0^{+}}}^{1} \bar{\omega}(0)=0, \\
\int_{0}^{1} \bar{\omega}(\eta) \mathrm{d} \eta+\int_{0}^{1} \mathcal{C}_{\mathfrak{D}_{0^{+}}}^{1} \bar{\omega}(\eta) \mathrm{d} \eta=\int_{0}^{1} \frac{(1-\eta)^{q_{2}-1}}{\Gamma\left(q_{2}\right)} \bar{\omega}(\eta) \mathrm{d} \eta,
\end{array}\right.
$$

respectively. Then, the following inequality is fulfilled:

$$
\|\omega-\bar{\omega}\|_{X} \leq \frac{E+E \sum_{m=1}^{\infty} \frac{\ell^{m}}{1-W-W \sum_{m=1}^{\infty} \frac{\ell_{1}^{m} \Gamma\left(m\left((\sigma-\delta)+q_{1}\right)+1\right)}{K_{1}^{m} \Gamma\left(m\left((\sigma-\delta)+q_{1}\right)+1\right)}}}{1-W\left(\ell^{m}\right.}
$$

so that $W+W \sum_{m=1}^{\infty} \frac{\ell^{m}}{K_{1}^{m} \Gamma\left(m\left((\sigma-\delta)+q_{1}\right)+1\right)}<1$, in which

$$
\begin{aligned}
E & =\frac{\left\|\hbar_{1}^{*}\right\|_{X}}{K_{1}}\left|\frac{1}{\Gamma\left(\sigma+q_{1}+1\right)}-\frac{1}{\Gamma\left((\sigma-\delta)+q_{1}+1\right)}\right| \\
& +\frac{\left\|\hbar_{1}^{*}\right\|_{X}}{\xi K_{1}}\left[\left|\frac{1}{\Gamma\left(\sigma+q_{2}+q_{1}+1\right)}-\frac{1}{\Gamma\left((\sigma-\delta)+q_{2}+q_{1}+1\right)}\right|\right. \\
& \left.+\left|\frac{1}{\Gamma\left(\sigma+q_{1}+2\right)}-\frac{1}{\Gamma\left((\sigma-\delta)+q_{1}+2\right)}\right|+\left|\frac{1}{\Gamma\left(\sigma+q_{1}+1\right)}-\frac{1}{\Gamma\left((\sigma-\delta)+q_{1}+1\right)}\right|\right]
\end{aligned}
$$

and

$$
W=\frac{1}{\xi K_{1}}\left(\frac{1}{\Gamma\left((\sigma-\delta)+q_{2}+q_{1}+1\right)}+\frac{1}{\Gamma\left((\sigma-\delta)+q_{1}+2\right)}+\frac{1}{\Gamma\left((\sigma-\delta)+q_{1}+1\right)}\right) \ell
$$

and $\left\|\hbar_{1}^{*}\right\|_{X}=\sup _{s \in[0,1]}\left|\hbar_{1}(s, \omega(s))\right|$.

Proof. First, obviously, Corollary 1 ensures the existence of solutions for two integrodifferential CFBVPs (9) and (12), and these solutions are given in the formats (10) and

$$
\begin{aligned}
\bar{\omega}(s)= & \frac{1}{K_{1}} \int_{0}^{s} \frac{(s-\eta)^{(\sigma-\delta)+q_{1}-1}}{\Gamma\left((\sigma-\delta)+q_{1}\right)} \hbar_{1}(\eta, \bar{\omega}(\eta)) \mathrm{d} \eta \\
& +\frac{s^{2}}{\xi K_{1}}\left[\int_{0}^{1} \frac{(1-\eta)^{(\sigma-\delta)+q_{2}+q_{1}-1}}{\Gamma\left((\sigma-\delta)+q_{2}+q_{1}\right)} \hbar_{1}(\eta, \bar{\omega}(\eta)) \mathrm{d} \eta\right. \\
& -\int_{0}^{1} \int_{0}^{\eta} \frac{(\eta-r)^{(\sigma-\delta)+q_{1}-1}}{\Gamma\left((\sigma-\delta)+q_{1}\right)} \hbar_{1}(r, \bar{\omega}(r)) \mathrm{d} r \mathrm{~d} \eta \\
& \left.-\int_{0}^{1} \int_{0}^{\eta} \frac{(\eta-r)^{(\sigma-\delta)+q_{1}-2}}{\Gamma\left((\sigma-\delta)+q_{1}-1\right)} \hbar_{1}(r, \bar{\omega}(r)) \mathrm{d} r \mathrm{~d} \eta\right],
\end{aligned}
$$


respectively. In this case, the following estimate for $\omega-\bar{\omega}$ is computed as

$$
\begin{aligned}
& |\omega(s)-\bar{\omega}(s)| \\
& \leq\left|\frac{1}{K_{1}} \int_{0}^{s}\left(\frac{(s-\eta)^{\sigma+q_{1}-1}}{\Gamma\left(\sigma+q_{1}\right)}-\frac{(s-\eta)^{(\sigma-\delta)+q_{1}-1}}{\Gamma\left((\sigma-\delta)+q_{1}\right)}\right) \hbar_{1}(\eta, \omega(\eta)) \mathrm{d} \eta\right| \\
& +\frac{1}{K_{1}} \int_{0}^{s} \frac{(s-\eta)^{(\sigma-\delta)+q_{1}-1}}{\Gamma\left((\sigma-\delta)+q_{1}\right)}\left|\hbar_{1}(\eta, \omega(\eta))-\hbar_{1}(\eta, \bar{\omega}(\eta))\right| \mathrm{d} \eta \\
& +\frac{s^{2}}{\xi K_{1}}\left[\left|\int_{0}^{1}\left(\frac{(1-\eta)^{\sigma+q_{2}+q_{1}-1}}{\Gamma\left(\sigma+q_{2}+q_{1}\right)}-\frac{(1-\eta)^{(\sigma-\delta)+q_{2}+q_{1}-1}}{\Gamma\left((\sigma-\delta)+q_{2}+q_{1}\right)}\right) \hbar_{1}(\eta, \omega(\eta)) \mathrm{d} \eta\right|\right. \\
& +\int_{0}^{1} \frac{(1-\eta)^{(\sigma-\delta)+q_{2}+q_{1}-1}}{\Gamma\left((\sigma-\delta)+q_{2}+q_{1}\right)}\left|\hbar_{1}(\eta, \omega(\eta))-\hbar_{1}(\eta, \bar{\omega}(\eta))\right| \mathrm{d} \eta \\
& +\left|\int_{0}^{1} \int_{0}^{\eta}\left(\frac{(\eta-r)^{\sigma+q_{1}-1}}{\Gamma\left(\sigma+q_{1}\right)}-\frac{(\eta-r)^{(\sigma-\delta)+q_{1}-1}}{\Gamma\left((\sigma-\delta)+q_{1}\right)}\right) \hbar_{1}(r, \omega(r)) \mathrm{d} r\right| \\
& +\int_{0}^{1} \int_{0}^{\eta} \frac{(\eta-r)^{(\sigma-\delta)+q_{1}-1}}{\Gamma\left((\sigma-\delta)+q_{1}\right)}\left|\hbar_{1}(r, \omega(r))-\hbar_{1}(r, \bar{\omega}(r))\right| \mathrm{d} r \\
& +\left|\int_{0}^{1} \int_{0}^{\eta}\left(\frac{(\eta-r)^{\sigma+q_{1}-2}}{\Gamma\left(\sigma+q_{1}-1\right)}-\frac{(\eta-r)^{(\sigma-\delta)+q_{1}-2}}{\Gamma\left((\sigma-\delta)+q_{1}-1\right)}\right) \hbar_{1}(r, \omega(r)) \mathrm{d} r\right| \\
& \left.+\int_{0}^{1} \int_{0}^{\eta} \frac{(\eta-r)^{(\sigma-\delta)+q_{1}-2}}{\Gamma\left((\sigma-\delta)+q_{1}-1\right)}\left|\hbar_{1}(r, \omega(r))-\hbar_{1}(r, \bar{\omega}(r))\right| \mathrm{d} r\right] \\
& \leq E+W\|\omega-\bar{\omega}\|_{X}+\int_{0}^{s} \frac{(s-\eta)^{(\sigma-\delta)+q_{1}-1}}{K_{1} \Gamma\left((\sigma-\delta)+q_{1}\right)} \ell|\omega(\eta)-\bar{\omega}(\eta)| \mathrm{d} \eta,
\end{aligned}
$$

where $E$ and $W$ are illustrated by (13) and (14). Therefore, by the generalized version of the Gronwall inequality (Theorem 4) and by letting $\hat{\omega}(s)=|\omega(s)-\bar{\omega}(s)|, u(s)=$ $E+W\|\omega-\bar{\omega}\|_{X}$ and $\psi(s)=\frac{\ell}{K_{1} \Gamma\left((\sigma-\delta)+q_{1}\right)}$, we reach

$$
\hat{\omega}(s) \leq u(s)+\psi(s) \int_{0}^{s}(s-\eta)^{(\sigma-\delta)+q_{1}-1} \hat{\omega}(\eta) \mathrm{d} \eta .
$$

The generalized version of the Gronwall inequality yields

$$
\hat{\omega}(s) \leq u(s)+\int_{0}^{s} \sum_{m=1}^{\infty}\left[\frac{\left(\psi(s) \Gamma\left((\sigma-\delta)+q_{1}\right)\right)^{m}}{\Gamma\left(m\left((\sigma-\delta)+q_{1}\right)\right)}(s-\eta)^{m\left((\sigma-\delta)+q_{1}\right)-1} u(\eta)\right] \mathrm{d} \eta,
$$

and so

$$
|\omega(s)-\bar{\omega}(s)| \leq E+W\|\omega-\bar{\omega}\|_{X}+\int_{0}^{s} \sum_{m=1}^{\infty}\left[\frac{\ell^{m}(s-\eta)^{m\left((\sigma-\delta)+q_{1}\right)-1}}{K_{1}^{m} \Gamma\left(m\left((\sigma-\delta)+q_{1}\right)\right)}\left(E+W\|\omega-\bar{\omega}\|_{X}\right)\right] \mathrm{d} \eta
$$


Consequently, we get

$$
\|\omega-\bar{\omega}\|_{X} \leq \frac{E+E \sum_{m=1}^{\infty} \frac{\ell^{m}}{1-W-W \sum_{m=1}^{\infty} \frac{\ell_{1}^{m}}{K_{1}^{m} \Gamma\left(m\left((\sigma-\delta)+q_{1}\right)+1\right)}}}{\left.K_{1}^{m} \Gamma\left((\sigma-\delta)+q_{1}\right)+1\right)}
$$

and this ends the argument.

\section{Some Examples for Simulation}

Here we aim to collect three examples for the simulation of the conditions of the above three theorems. With the help of these special numerical examples, we validate theoretical outcomes according to the methods implemented in the theorems.

Example 1. (For illustrating Theorem 5) With regard to the designed construction for the nonlinear integro-differential CFBVP (1), we here suggest the following special FBVP as

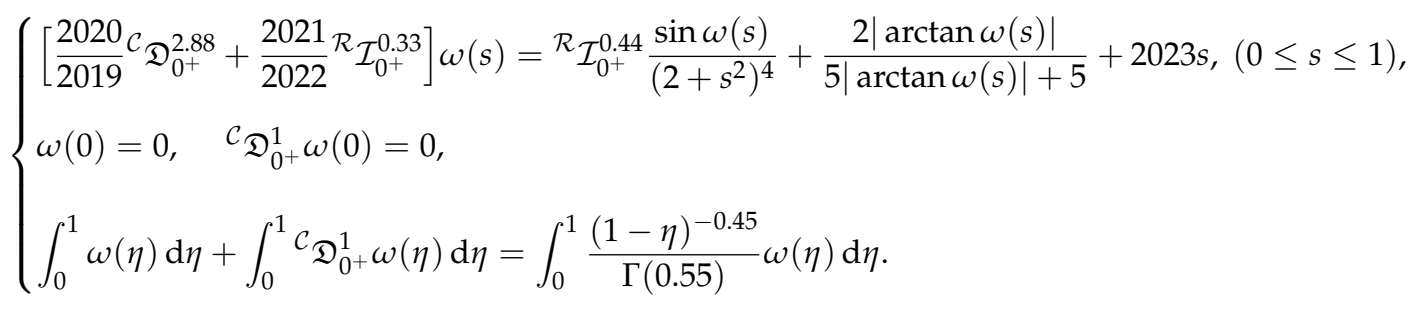

The parameters $K_{1}=\frac{2020}{2019}, K_{2}=\frac{2021}{2020}, \sigma=2.88, q_{1}=0.44, q_{2}=0.55$, and $\theta=0.33$ are supposed by considering the above suggested CFBVP. In addition to these, continuous functions $\hbar_{1}, \hbar_{2}:[0,1] \times \mathbb{R} \rightarrow \mathbb{R}$ are regarded as

$$
\hbar_{1}(s, \omega(s))=\frac{\sin \omega(s)}{(4+s)^{2}}, \quad \hbar_{2}(s, \omega(s))=\frac{2|\arctan \omega(s)|}{5|\arctan \omega(s)|+5}+2023 s .
$$

It is considered that for any $\omega_{1}, \omega_{2} \in \mathbb{R}$, we have

$$
\begin{aligned}
\left|\hbar_{2}\left(s, \omega_{1}\right)-\hbar_{2}\left(s, \omega_{2}\right)\right| & \leq\left|\frac{2\left|\arctan \omega_{1}(s)\right|}{5\left|\arctan \omega_{1}(s)\right|+5}-\frac{2\left|\arctan \omega_{2}(s)\right|}{5\left|\arctan \omega_{2}(s)\right|+5}\right| \\
& \leq \frac{2}{5}\left|\arctan \omega_{1}(s)-\arctan \omega_{2}(s)\right| \\
& \leq \frac{2}{5}\left|\omega_{1}(s)-\omega_{2}(s)\right| .
\end{aligned}
$$

Hence we get $\left|\hbar_{2}\left(s, \omega_{1}\right)-\hbar_{2}\left(s, \omega_{2}\right)\right| \leq \frac{2}{5}\left|\omega_{1}(s)-\omega_{2}(s)\right|$ and it is found out that $\hat{L}^{*}=$ $\frac{2}{5}>0$. On the other side, a continuous function $\Phi(s)=\frac{1}{\left(2+s^{2}\right)^{4}}$ exists on $[0,1]$ provided that an inequality $\left|\hbar_{1}(s, \omega(s))\right| \leq\left|\frac{\sin \omega(s)}{(4+s)^{2}}\right| \leq \Phi(s)$ is valid for all $\omega \in \mathbb{R}$. In that phase, we obtain $\|\Phi\|=\sup _{s \in[0,1]} \Phi(s)=0.0625$. With regard to the above parameters, it can be quickly obtained that $\xi \approx 0.7641, \tilde{\Delta}^{(1)} \approx 0.4107, \tilde{\Delta}^{(2)} \approx 0.6353$, and $\tilde{\Delta}^{(3)} \approx 0.3532$. Accordingly,

$$
\tilde{\Delta}^{(1)}+\hat{L}^{*} \tilde{\Delta}^{(2)} \approx 0.6648<1 .
$$


It can be concluded that all assertions of Theorem 5 hold for the suggested CFBVP (15). As a result, the statement of Theorem 5 reveals that the nonlinear integro-differential CFBVP (15) contains a solution on $[0,1]$.

Example 2. (For illustrating Theorem 6) With regard to the designed construction for the nonlinear integro-differential CFBVP (1), we here suggest another special CFBVP as

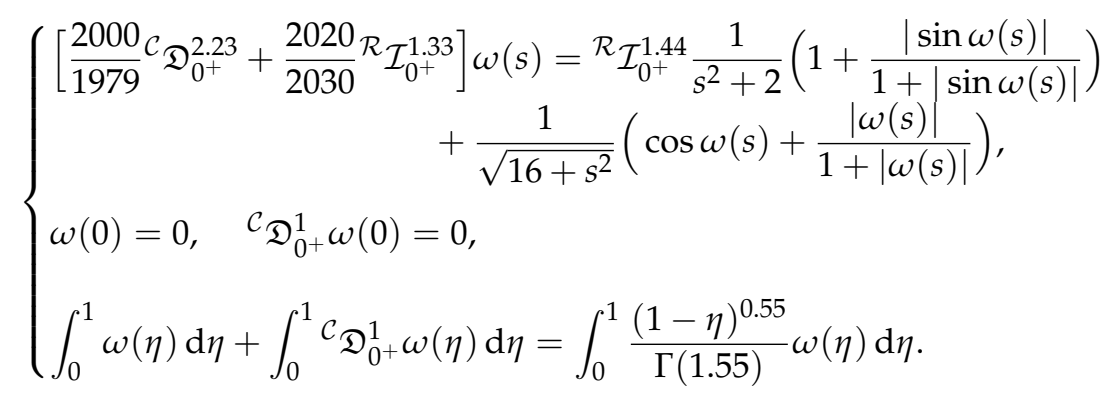

The parameters $K_{1}=\frac{2020}{1979}, K_{2}=\frac{2020}{2030}, \sigma=2.23, q_{1}=1.44, q_{2}=1.55$, and $\theta=1.33$ are supposed according to the above suggested CFBVP (16). Along with these, the functions $\hbar_{1}, \hbar_{2}:[0,1] \times \mathbb{R} \rightarrow \mathbb{R}$ illustrated as

$$
\hbar_{1}(s, \omega(s))=\frac{1}{s^{2}+2}\left(1+\frac{|\sin \omega(s)|}{1+|\sin \omega(s)|}\right)
$$

and

$$
\hbar_{2}(s, \omega(s))=\frac{1}{\sqrt{16+s^{2}}}\left(\frac{|\omega(s)|}{1+|\omega(s)|}+\cos \omega(s)\right)
$$

are continuous on $[0,1]$. Apparently, the following inequalities hold

$$
\left|\hbar_{1}(s, \omega(s))\right| \leq \frac{1}{s^{2}+2}(1+\|\omega\|), \quad\left|\hbar_{2}(s, \omega(s))\right| \leq \frac{1}{\sqrt{16+s^{2}}}(1+\|\omega\|) .
$$

Set $Y_{1}(s)=\frac{1}{\sqrt{16+s^{2}}}$ and $Y_{2}(s)=\frac{1}{s^{2}+2}$ and $\mathfrak{N}_{1}(\|\omega\|)=\mathfrak{N}_{2}(\|\omega\|)=1+\|\omega\|$. Note that $\left\|\mathrm{Y}_{1}\right\|=\frac{1}{4}=0.25,\left\|\mathrm{Y}_{2}\right\|=\frac{1}{2}=0.5$ and $\mathfrak{N}_{1}(\varepsilon)=\mathfrak{N}_{2}(\varepsilon)=1+\varepsilon$. By invoking the above parameters, we reach $\xi \approx 1.1730, \tilde{\Delta}^{(1)} \approx 0.1646<1, \tilde{\Delta}^{(2)} \approx 0.8870$, and $\tilde{\Delta}^{(3)} \approx 0.1410$. Therefore, in the light of $(\mathbb{C} 4)$, we arrive at $\varepsilon>2.3359$. We find that all statements of Theorem 6 are valid for the CFBVP (16). Consequently, with regard to Theorem 6, the nonlinear integro-differential CFBVP (16) contains a solution on $[0,1]$.

Example 3. (For illustrating Theorem 7) With regard to the designed construction for the nonlinear integro-differential CFBVP (1), we prepare the third special CFBVP as

$$
\left\{\begin{array}{l}
{\left[0.9^{\mathcal{C}} \mathfrak{D}_{0^{+}}^{2.44}+0.5^{\mathcal{R}} \mathcal{I}_{0^{+}}^{2.33}\right] \omega(s)={ }^{\mathcal{R}} \mathcal{I}_{0^{+}}^{3.44}\left(\exp \left(s^{2}\right)+\frac{|\omega(s)|}{2+2|\omega(s)|}\right)+\frac{|\sin (s)||\omega(s)|}{4|\omega(s)|+4},} \\
\omega(0)=0, \quad \mathcal{C}_{\mathfrak{D}^{+}}^{1} \omega(0)=0 \\
\int_{0}^{1} \omega(\eta) \mathrm{d} \eta+\int_{0}^{1} \mathcal{C}_{\mathfrak{D}_{0^{+}}}^{1} \omega(\eta) \mathrm{d} \eta=\int_{0}^{1} \frac{(1-\eta)^{1.55}}{\Gamma(2.55)} \omega(\eta) \mathrm{d} \eta .
\end{array}\right.
$$


The parameters $K_{1}=0.9, K_{2}=0.5, \sigma=2.44, q_{1}=3.44, q_{2}=2.55$, and $\theta=2.33$ are supposed according to the above suggested CFBVP (17). Besides, functions $\hbar_{1}, \hbar_{2}:[0,1] \times \mathbb{R} \rightarrow \mathbb{R}$ displayed as

$$
\hbar_{1}(s, \omega(s))=\exp \left(s^{2}\right)+\frac{|\omega(s)|}{2+2|\omega(s)|}, \quad \hbar_{2}(s, \omega(s))=\frac{|\sin (s)||\omega(s)|}{4|\omega(s)|+4}
$$

are assumed to be continuous on the relevant domain $[0,1]$. Then we arrive at values $\hat{L}^{*}=0.25$ and $\mathfrak{R}=0.5$, since we can easily compute that

$$
\left|\hbar_{1}\left(s, \omega_{1}(1)\right)-\hbar_{2}\left(s, \omega_{2}(s)\right)\right| \leq 0.5\left(\left|\omega_{1}(s)-\omega_{2}(s)\right|\right)
$$

and

$$
\left|\hbar_{2}\left(s, \omega_{1}(s)\right)-\omega_{2}\left(s, \omega_{2}(s)\right)\right| \leq 0.25\left(\left|\omega_{1}(s)-\omega_{2}(s)\right|\right) .
$$

Finally, by taking into account the above constants, we find that $\xi \approx 1.2981$ and

$$
\tilde{\Delta}^{(1)}+\hat{L}^{*} \tilde{\Delta}^{(2)}+\mathfrak{R} \tilde{\Delta}^{(3)} \approx 0.1848<1 .
$$

Accordingly, we comprehend that all statements of Theorem 7 are satisfied for the aforesaid CFBVP (17). In conclusion, with regard to Theorem 7 , the nonlinear integro-differential CFBVP (17) possesses a solution on the domain $[0,1]$.

\section{Conclusions}

In the current research, we designed a general boundary value problem in the Caputo fractional integro-differential setting (1) with two different orders $\theta$ and $q_{1}$. In addition, we considered mixed integro-derivative boundary conditions which include general and new structures, and the right-hand side of the equation is considered a sum of two nonlinear terms. It is obvious that if we take $K_{2}=0$ and $\hbar_{2} \equiv 0$, then our problem is transformed into the standard integro-differential equation

$$
\left\{\begin{array}{l}
\mathcal{C}_{\mathfrak{D}_{0^{+}}^{\sigma}} \omega(s)=\frac{1}{K_{1}} \mathcal{R}_{\mathcal{I}_{0^{+}}}^{q_{1}} \hbar_{1}(s, \omega(s)), \quad(0 \leq s \leq 1), \\
\omega(0)=0, \quad \mathcal{C}_{\mathfrak{D}_{0^{+}}^{1}}^{1} \omega(0)=0, \\
\int_{0}^{1} \omega(\eta) \mathrm{d} \eta+\int_{0}^{1} \mathcal{C}_{\mathfrak{D}_{0^{+}}^{1}}^{1} \omega(\eta) \mathrm{d} \eta=\int_{0}^{1} \frac{(1-\eta)^{q_{2}-1}}{\Gamma\left(q_{2}\right)} \omega(\eta) \mathrm{d} \eta
\end{array}\right.
$$

To be more specific, if we take $\theta=q_{1}=0, K_{2}=1$, and $\hbar_{2} \equiv 0$, then for $0<$ $\mu<1$, we have the fractional pantograph differential equation $\mathcal{C}_{\mathfrak{D}_{0^{+}}^{\sigma}} \omega(s)=-\frac{1}{K_{1}} \omega(s)+$ $\frac{1}{K_{1}} \hbar_{1}(s, \omega(\mu s))$ with integral boundary conditions given by (1). On the other side, by assuming $\theta=0$ and $\hbar_{2} \equiv 0$, a simple form of the fractional Langevin integro-differential equation is derived as $\left[K_{1} \mathcal{C}_{\mathfrak{D}_{0^{+}}^{\sigma}}+K_{2}\right] \omega(s)={ }^{\mathcal{R}} \mathcal{I}_{0^{+}}^{q_{1}} \hbar_{1}(s, \omega(s))$. By some direct computations, we derived an integral equation corresponding to the given CFBVP and investigated existence results for solutions of the given problem (1) in three distinct cases. Indeed, in the two first cases, we proved the existence results by means of the Krasnosel'skii and Leray-Schauder fixed point theorems, and in the third case, we checked the uniqueness of solutions with the aid of the Banach contraction principle. Additionally, by considering the generalized version of the Gronwall inequality, the dependence of solutions for a special case of the integro-differential CFBVP (1) is investigated. In the final step, we prepared three simulation examples to guarantee the applicability of the theoretical outcomes.

Author Contributions: Conceptualization, S.R., S.K.N., S.E., and J.T.; methodology, S.R., S.K.N., A.A., S.E., and J.T.; formal analysis, S.R., S.K.N., A.A., S.E., and J.T.; funding acquisition, J.T. All authors have read and agreed to the published version of the manuscript. 
Funding: This research was funded by King Mongkut's University of Technology North Bangkok. Contract no. KMUTNB-62-KNOW-28.

Conflicts of Interest: The authors declare no conflict of interest.

\section{References}

1. Debnath, L. Recent applications of fractional calculus to science and engineering. Int. J. Math. Math. Sci. 2003, 54, 3413-3442. [CrossRef]

2. Hilfer, R. Applications of Fractional Calculus in Physics; World Scientific: Singapore, 2000.

3. Kempfle, S.; Schafer, I.; Beyer, H. Fractional calculus via functional calculus: Theory and applications. Nonlinear Dyn. 2002, 29, 99-127. [CrossRef]

4. Kilbas, A.A.; Srivastava, H.M.; Trujillo, J.J. Theory and Applications of Fractional Differential Equations; North Holland Mathematics Studies: Amsterdam, The Netherlands, 2006; Volume 203.

5. Baleanu, D.; Etemad, S.; Rezapour, S. A hybrid Caputo fractional modeling for thermostat with hybrid boundary value conditions. Bound. Value Probl. 2020, 1, 64. [CrossRef]

6. Goswami, A.; Singh, J.; Kumar, D.; Sushila. An efficient analytical approach for fractional equal width equations describing hydro-magnetic waves in cold plasma. Phys. A Stat. Mech. Appl. 2019, 524, 563-575. [CrossRef]

7. Magin, R. Fractional calculus models of complex dynamics in biological tissues. Comput. Math. Appl. 2010, 59, 1586-1593. [CrossRef]

8. Merala, F.C.; Roystona, T.J.; Magin, R. Fractional calculus in viscoelasticity: An experimental study. Commun. Nonlinear Sci. Numer. Simul. 2010, 15, 939-945. [CrossRef]

9. Oustaloup, A.; Pommier, V.; Lanusse, P. Design of a fractional control using performance contours: Application to an electromechanical system. Fract. Calc. Appl. Anal. 2003, 6, 1-24.

10. Rezapour, S.; Mohammadi, H.; Jajarmi, A. A new mathematical model for Zika virus transmission. Adv. Differ. Equ. 2020, 2020, 589. [CrossRef]

11. Ullah, S.; Khan, M.A.; Farooq, M.; Hammouch, Z.; Baleanu, D. A fractional model for the dynamics of tuberculosis infection using Caputo-Fabrizio derivative. Discret. Contin. Dyn. Syst. 2020, 13, 975-993. [CrossRef]

12. $\mathrm{Xu}, \mathrm{H}$. Analytical approximations for a population growth model with fractional order. Commun. Nonlinear Sci. Numer. Simul. 2009, 4, 1978-1983. [CrossRef]

13. Abdo, M.S.; Shah, K.; Panchal, S.K.; Wahash, H.A. Existence and Ulam stability results of a coupled system for terminal value problems involving $\psi$-Hilfer fractional operator. Adv. Differ. Equ. 2020, 2020, 316. [CrossRef]

14. Abdo, M.S.; Thabet, S.T.M.; Ahmad, B. The existence and Ulam-Hyers stability results for $\psi$-Hilfer fractional integro-differential equations. J. Pseudo-Differ. Oper. Appl. 2020, 11, 1757-1780. [CrossRef]

15. Alsaedi, A.; Ahmad, B.; Alghanmi, M.; Ntouyas, S.K. On a generalized Langevin type nonlocal fractional integral multivalued problem. Mathematics 2019, 7, 1015. [CrossRef]

16. Amara, A. Existence results for hybrid fractional differential equations with three-point boundary conditions. AIMS Math. 2020, 5 , 1074-1088. [CrossRef]

17. Ben Chikh, S.; Amara, A.; Etemad, S.; Rezapour, S. On Hyers-Ulam stability of a multi-order boundary value problems via Riemann-Liouville derivatives and integrals. Adv. Differ. Equ. 2020, 2020, 547. [CrossRef]

18. Ben Chikh, S.; Amara, A.; Etemad, S.; Rezapour, S. On Ulam-Hyers-Rassias stability of a generalized Caputo type multi-order boundary value problems with four-point mixed integro-derivative conditions. Adv. Differ. Equ. 2020, 2020, 680. [CrossRef]

19. Cui, Y.; Ma, W.; Sun, Q.; Su, X. New uniqueness results for boundary value problem of fractional differential equation. Nonlinear Anal. Model. Control 2018, 23, 31-39. [CrossRef]

20. Etemad, S.; Ntouyas, S.K.; Tariboon, J. Existence results for three-point boundary value problems for nonlinear fractional differential equations. J. Nonlinear Sci. Appl. 2016, 9, 2105-2116. [CrossRef]

21. Kheiryan, A.; Rezapour, S. A multi-singular fractional equation and the Hyers-Ulam stability. Int. J. Appl. Comput. Math. 2020, 6, 155. [CrossRef]

22. Mohammadi, H.; Rezapour, S.; Etemad, S. On a hybrid fractional Caputo-Hadamard boundary value problem with hybrid Hadamard integral boundary value conditions. Adv. Differ. Equ. 2020, 2020, 455. [CrossRef]

23. Peng, L.; Zhou, Y. Bifurcation from interval and positive solutions of the three-point boundary value problem for fractional differential equations. Appl. Math. Comput. 2015, 257, 458-466. [CrossRef]

24. Thabet, S.T.M.; Etemad, S.; Rezapour, S. On a new structure of the pantograph inclusion problem in the Caputo conformable setting. Bound. Value Probl. 2020, 2020, 171. [CrossRef]

25. Wang, G.; Pei, K.; Agarwal, R.P.; Zhang, L.; Ahmad, B. Nonlocal Hadamard fractional boundary value problem with Hadamard integral and discrete boundary conditions on a half-line. J. Comput. Appl. Math. 2018, 343, 230-239. [CrossRef]

26. Davies, P.J.; Duncan, D.B. Numerical approximation of first kind Volterra convolution integral equations with discontinuous kernels. J. Integral Equ. Appl. 2017, 29, 41-73. [CrossRef]

27. Ahmad, B.; Broom, A.; Alsaedi, A.; Ntouyas, S.K. Nonlinear integro-differential equations involving mixed right and left fractional derivatives and integrals with nonlocal boundary data. Mathematics 2020, 8, 336. [CrossRef] 
28. Ahmad, B.; Luca, R. Existence of solutions for sequential fractional integro-differential equations and inclusions with nonlocal boundary conditions. Appl. Math. Comput. 2018, 339, 516-534. [CrossRef]

29. Baleanu, D.; Etemad, S.; Rezapour, S. On a fractional hybrid integro-differential equation with mixed hybrid integral boundary value conditions by using three operators. Alex. Eng. J. 2020. [CrossRef]

30. Etemad, S.; Ntouyas, S.K.; Ahmad, B. Existence theory for a fractional $q$-integro-difference equation with $q$-integral boundary conditions of different orders. Mathematics 2019, 7, 659. [CrossRef]

31. Jamil, M.; Khan, R.A.; Shah, K. Existence theory to a class of boundary value problems of hybrid fractional sequential integrodifferential equations. Bound. Value Probl. 2019, 2019, 77. [CrossRef]

32. Phuong, N.D.; Sakar, F.M.; Etemad, S.; Rezapour, S. A novel fractional structure of a multi-order quantum multi-integro-differential problem. Adv. Differ. Equ. 2020, 2020, 633. [CrossRef]

33. Yang, A.M.; Han, Y.; Zhang, Y.Z.; Wang, L.T.; Zhang, D.; Yang, X.J. On nonlocal fractional Volterra integro-differential equations in fractional steady heat transfer. Therm. Sci. 2016, 20, S789-S793. [CrossRef]

34. Zhao, Y.; Huang, L.; Wang, X.; Zhu, X. Existence of solutions for fractional integro-differential equation with multipoint boundary value problem in Banach spaces. Abstr. Appl. Anal. 2012, 2012, 172963. [CrossRef]

35. Ahmad, B.; Ntouyas, S.K.; Tariboon, J. On hybrid Caputo fractional integro-differential inclusions with nonlocal conditions. J. Nonlinear Sci. Appl. 2016, 9, 4235-4246. [CrossRef]

36. Bazgir, H.; Ghazanfari, B. Existence of solutions for fractional integro-differential equations with non-local boundary conditions. Math. Comput. Appl. 2018, 23, 36. [CrossRef]

37. Podlubny, I. Fractional Differential Equations; Academic Press: New York, NY, USA, 1999.

38. Samko, S.G.; Kilbas, A.A.; Marichev, O. Fractional Integrals and Derivatives: Theory and Applications; Gordon and Breach: Amsterdam, The Netherlands, 1993.

39. Miller, S.; Ross, B. An Introduction to the Fractional Calculus and Fractional Differential Equations; John Wiley: Hoboken, HJ, USA, 1993.

40. Krasnoselskii, M.A. Two remarks on the method of successive approximations. Usp. Mat. Nauk. 1955, 10, $123-127$.

41. Granas, A.; Dugundji, J. Fixed Point Theory; Springer: New York, NY, USA, 2003.

42. Deimling, K. Nonlinear Functional Analysis; Springer: New York, NY, USA, 1985.

43. Ye, H.; Gao, J.; Ding, Y. A generalized Gronwall inequity and its application to a fractional differential equation. J. Math. Anal. Appl. 2007, 328, 1075-1081. [CrossRef]

44. Apartsyn, A.S. Nonclassical Linear Volterra Equations of the First Kind; Inverse and Ill-Posed Problems, Series 39; De Gruyter: Berlin, Germany, 2003. 